\title{
Computable exact bounds for linear outputs from stabilized solutions of the advection-diffusion-reaction equation*
}

\author{
Núria Parés, Pedro Díez and Antonio Huerta \\ Laboratori de Càlcul Numèric (LaCàN), Universitat Politècnica de Catalunya, Barcelona, Spain \\ e-mail:\{nuria.pares,pedro.diez,antonio.huerta\}@upc.edu.
}

\begin{abstract}
SUMMARY
The paper introduces a methodology to compute strict upper and lower bounds for linear-functional outputs of the exact solutions of the advection-reaction-diffusion equation. The bounds are computed using implicit a-posteriori error estimators from stabilized finite element approximations of the exact solution. A new methodology is introduced, based in the ideas presented in [1] for the Galerkin formulation, that allows obtaining bounds also for stabilized formulations. This methodology is combined with both hybrid-flux and flux-free techniques for error assessment. The application to stabilized formulations provides sharper estimates than when applied to Galerkin methods. The best results are found in combination with the fluxfree technique.
\end{abstract}

KEY WORDS: Linear-functional outputs; Exact/guaranteed/strict bounds; Stabilization methods; Error estimation; Goal-oriented adaptivity; Advection-reaction-diffusion equation.

\section{INTRODUCTION}

The certification of numerical simulations of partial differential equations is fundamental in many engineering applications, where end-users aim at obtaining an approximation of a specific magnitude extracted from the global solution (quantity of interest) with a prescribed accuracy.

Since the mid 2000s, attention has been devoted to provide certified bounds for quantities of interest $[2,3,4,5,6,7,8,9]$. In particular [1] presents a comparison of the performance of two of the main techniques to compute guaranteed bounds for quantities of interest in the context of the advection-reaction-diffusion equation: a standard residual type estimator (hybrid-flux) proposed in [10] and the new flux-free technique proposed in [11].

For advection dominated problems, the use of stabilized formulations [12] is of utmost importance, since Galerkin approximations are often corrupted by spurious node-to-node oscillations. In the present paper we develop an extension of the techniques presented in [1] to compute guaranteed bounds for quantities of interest from stabilized approximations of the exact solution. Thus, strict bounds for quantities of interest are obtained using implicit residual error estimates, both using hybrid-flux techniques $[13,14]$ and the flux-free technique first devised in [11]. 


\section{PROBLEM STATEMENT}

\subsection{Model problem}

The steady advection-reaction-diffusion equation reads

$$
\begin{aligned}
-\nabla \cdot(\nu \nabla u)+\boldsymbol{\alpha} \cdot \boldsymbol{\nabla} u+\sigma u=f & \text { in } \Omega, \\
u=u_{D} & \text { on } \Gamma_{\mathrm{D}}, \\
\nu \boldsymbol{\nabla} u \cdot \boldsymbol{n}=g & \text { on } \Gamma_{\mathrm{N}},
\end{aligned}
$$

where $\Omega$ is a plane polygonal domain whose boundary $\partial \Omega$ is partitioned into two disjoint sets $\Gamma_{\mathrm{D}}$ (of nonzero measure) and $\Gamma_{N}$, and $\boldsymbol{n}$ is the outward unit normal vector to $\partial \Omega$. The datum $\nu$ is assumed to be strictly positive and $\sigma$ is assumed to be non-negative.

The standard variational formulation of the problem consists of finding $u \in \mathcal{U}$ such that

$$
a(u, v)=\ell(v) \quad \forall v \in v,
$$

where $a(\cdot, \cdot): \mathcal{H}^{1}(\Omega) \times \mathcal{H}^{1}(\Omega) \rightarrow \mathbb{R}$ and $\ell: \mathcal{H}^{1}(\Omega) \rightarrow \mathbb{R}$ denote the bilinear and linear forms respectively defined by

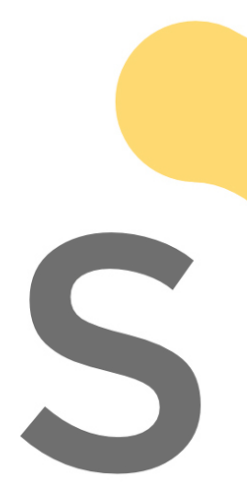

\section{r}

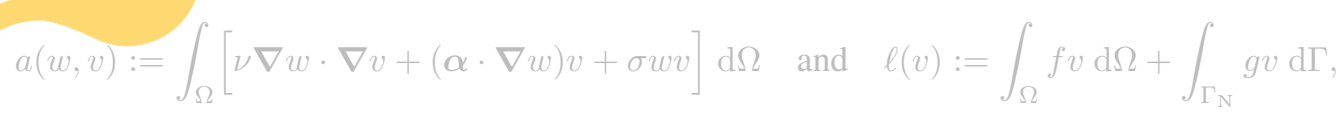

and $\mathcal{U}:=\left\{v \in \mathcal{H}^{1}(\Omega),\left.v\right|_{\Gamma_{\mathrm{D}}}=u_{\mathrm{D}}\right\}$ and $\mathcal{\nu}:=\left\{v \in \mathcal{H}^{1}(\Omega),\left.v\right|_{\Gamma_{\mathrm{D}}}=0\right\}$ are the solution and test

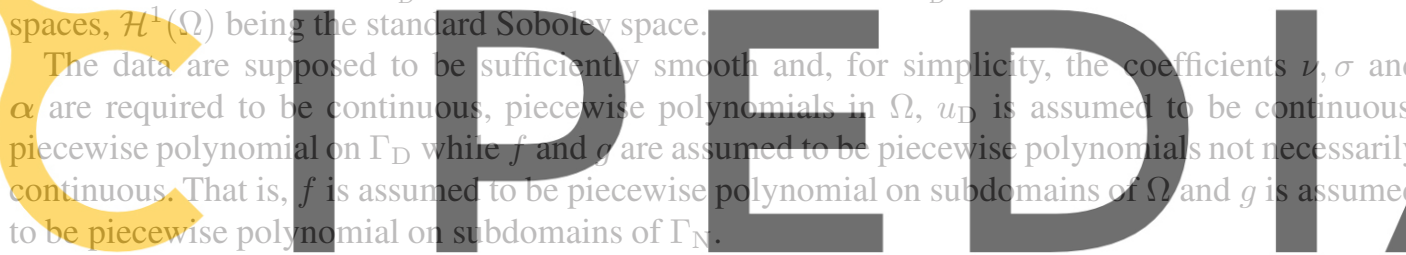

o be piecewise polynomial on stbdomains of 1

The nonsymmetric bilinear form $a(\cdot, \cdot)$ is continuous and coercive in $\mathcal{V}$. In order to ensure that, it

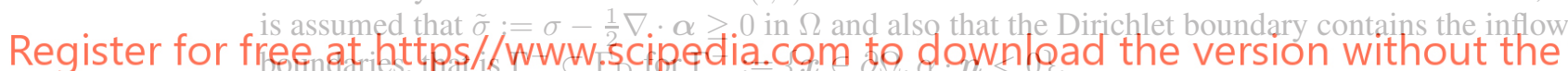

\subsection{Stabilized finite element approximation}

Various stabilization techniques are available for advection-reaction-diffusion problems, all aiming at precluding oscillations of the finite element approximations without requiring severe mesh refinement [12]. However, in view of the developments in section 4, the streamline upwind PetrovGalerkin method (SUPG) is adopted in this work (see remark 1 for other possibilities).

The so-called SUPG finite element method is described using a triangulation of the computational domain $\Omega$ into $n_{\mathrm{el}}$ triangles where $\Omega_{k}$ denotes a general triangle, $k=1, \ldots, n_{\mathrm{el}}$, and the finitedimensional spaces $\mathcal{U}^{h} \subset \mathcal{U}$ and $\mathcal{V}^{h} \subset \mathcal{V}$ consisting of the usual continuous, piecewise-polynomial functions of degree $p \geq 1$.

Then, for a given choice of the stabilization parameter to be specified, an approximation of the true solution $u$ is obtained by seeking $u_{h} \in \mathcal{U}^{h}$ such that

$$
a\left(u_{h}, v\right)+\sum_{k=1}^{n_{\mathrm{el}}} \int_{\Omega_{k}} \tau_{k}^{P} \mathcal{R}^{P}\left(u_{h}\right) \boldsymbol{\alpha} \cdot \nabla v d \Omega=\ell(v) \quad \forall v \in \mathcal{V}^{h},
$$

where

$$
\mathcal{R}^{P}(w)=-\nabla \cdot(\nu \nabla w)+\boldsymbol{\alpha} \cdot \nabla w+\sigma w-f
$$

denotes the strong residual of the differential equation (1a) and $\tau_{k}^{P}$ is the local stabilization parameter associated with element $\Omega_{k}$. Note that the superscript $P$ is used to denote quantities related with the original problem described by equations (1) or (3). 


\section{Remark 1}

Although all the developments herein concern the SUPG method, the presented theory is also valid, as it stands, for other stabilization techniques of the form

$$
a\left(u_{h}, v\right)+\sum_{k=1}^{n_{\mathrm{el}}} \int_{\Omega_{k}} \tau_{k}^{P} \mathcal{P}\left(u_{h}\right) \boldsymbol{\alpha} \cdot \nabla v d \Omega=\ell(v) \quad \forall v \in \mathcal{V}^{h},
$$

where $\mathcal{P}(\cdot)$ is a certain given operator. Two widely used choices are $\mathcal{P}=\mathcal{R}^{P}$, which yields the aforementioned consistent SUPG method, and $\mathcal{P}(v)=\boldsymbol{\alpha} \cdot \boldsymbol{\nabla} v$, which yields the streamline-upwind (SU) method. Note that although the present work covers some widely used stabilization techniques, it does not cover the full spectrum of stabilization techniques. For instance, the only consistent stabilization technique covered by this approach is the SUPG method, and thus for instance the Galerkin-least-squares (GLS) method is beyond the scope of the work. Other specific techniques should be developed to broaden the extent of the work.

\subsection{Goal oriented simulations: Outputs and adjoint problem}

The purpose of the present work is to develop a posteriori error estimators providing computable bounds for a given quantity of interest (also called output) and giving local error indicators. The local information is used to drive adaptive refinement procedures. The final aim is to achieve the prescribed aecuracy in the approximations of the quantities of interest.

When it comes to goal-oriented error estimation, controlling a global measure of the error in the field solution $u$ is not necessarily relevant. In this case, the interest is placed in certifying the accuracy of the desired output of the simulation, which depends on $u$, and is denoted by $s:=\ell^{\mathcal{O}}(u)$.
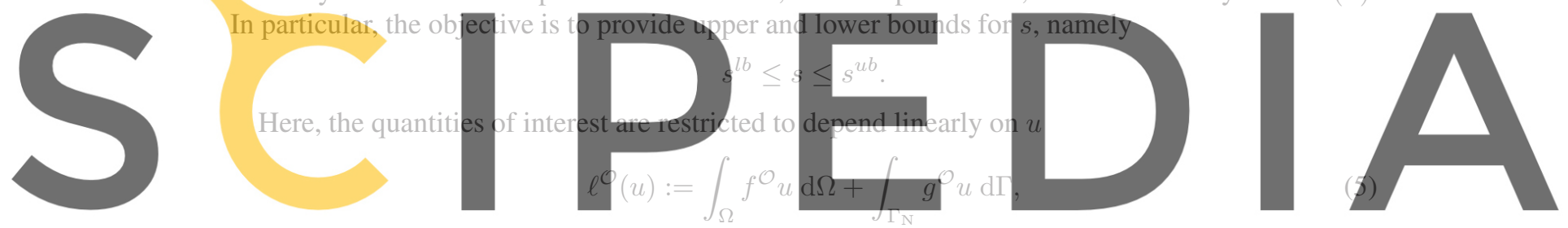

but other quantities of interest may also be considered [1, 4, 15]. That data $f^{\mathcal{O}}$ is assumed to

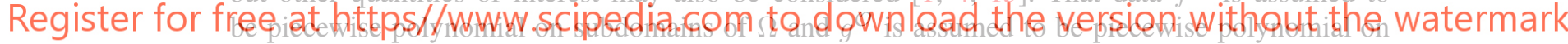
subdomains of $\Gamma_{\mathrm{N}}$.

One of the key ingredients in developing strategies to compute bounds for the output $s$ is the definition of an auxiliary problem, denoted adjoint problem $[1,10,16,14,17]$. The variational form of the adjoint problem consists of finding $\psi \in \mathcal{V}$ such that

$$
a(v, \psi)=\ell^{\mathcal{O}}(v) \quad \forall v \in \mathcal{V},
$$

which is equivalent to determine $\psi$ such that

$$
\begin{aligned}
-\nabla \cdot(\nu \nabla \psi)-\boldsymbol{\alpha} \cdot \boldsymbol{\nabla} \psi+(\sigma-\nabla \cdot \boldsymbol{\alpha}) \psi=f^{\mathcal{O}} & \text { in } \Omega, \\
\psi=0 & \text { on } \Gamma_{\mathrm{D}}, \\
\nu \boldsymbol{\nabla} \psi \cdot \boldsymbol{n}+\boldsymbol{\alpha} \cdot \boldsymbol{n} \psi=g^{\mathcal{O}} & \text { on } \Gamma_{\mathrm{N}} .
\end{aligned}
$$

Analogous to the direct (or primal) problem, the adjoint problem is solved numerically using the SUPG method. Thus, $\psi_{h} \in \mathcal{V}^{h}$ is such that

$$
a\left(v, \psi_{h}\right)-\sum_{k=1}^{n_{\mathrm{el}}} \int_{\Omega_{k}} \tau_{k}^{D} \mathcal{R}^{D}\left(\psi_{h}\right) \boldsymbol{\alpha} \cdot \nabla v d \Omega=\ell^{\mathcal{O}}(v) \quad \forall v \in \mathcal{V}^{h},
$$

where

$$
\mathcal{R}^{D}(w)=-\nabla \cdot(\nu \nabla w)-\boldsymbol{\alpha} \cdot \boldsymbol{\nabla} w+(\sigma-\nabla \cdot \boldsymbol{\alpha}) w-f^{\mathcal{O}}
$$

is the strong residual of the differential equation (6a) and $\tau_{k}^{D}$ is the stabilization parameter associated with the adjoint problem and the element $\Omega_{k}$. The choice of the stabilization parameter both for the primal and adjoint problem is addressed in section 6. 


\section{ENERGY REFORMULATION: REPRESENTATION OF THE OUTPUT BOUNDS}

Bounds for the quantity of interest $s=\ell^{\mathcal{O}}(u)$ can be recovered from standard Galerkin approximations of the primal and adjoint problems using the well-known inequality

$$
\ell^{\mathcal{O}}\left(u_{h}\right)-\frac{1}{2}\left\|\kappa e^{\mathrm{s}}-\frac{1}{\kappa} \varepsilon^{\mathrm{s}}\right\|^{2} \leq \ell^{\mathcal{O}}(u) \leq \ell^{\mathcal{O}}\left(u_{h}\right)+\frac{1}{2}\left\|\kappa e^{\mathrm{s}}+\frac{1}{\kappa} \varepsilon^{\mathrm{s}}\right\|^{2},
$$

where $\|\cdot\|$ is the energy norm induced by the symmetric counterpart of the bilinear form $a(\cdot, \cdot)$, $e^{\mathrm{S}}$ and $\varepsilon^{\mathrm{s}} \in \mathcal{V}$ are the solutions of the symmetrized residual equations and $\kappa \in \mathbb{R}$ is an arbitrary non-zero scalar parameter $[14,10,1]$.

To be specific, let $a^{\mathrm{s}}(v, w):=(a(w, v)+a(v, w)) / 2$ be the symmetric counterpart of $a(\cdot, \cdot)$. Then, $\|v\|^{2}=a^{\mathrm{s}}(v, v)=a(v, v)$ is generally referred to as the energy norm, and $e^{\mathrm{s}} \in \mathcal{V}$ and $\varepsilon^{\mathrm{s}} \in \mathcal{V}$, which are often dubbed as symmetric primal and adjoint errors, are the solution of the residual equations

$$
a^{\mathrm{s}}\left(e^{\mathrm{s}}, v\right)=\ell(v)-a\left(u_{h}, v\right)=: R^{\mathrm{P}}(v) \quad \forall v \in \nu,
$$

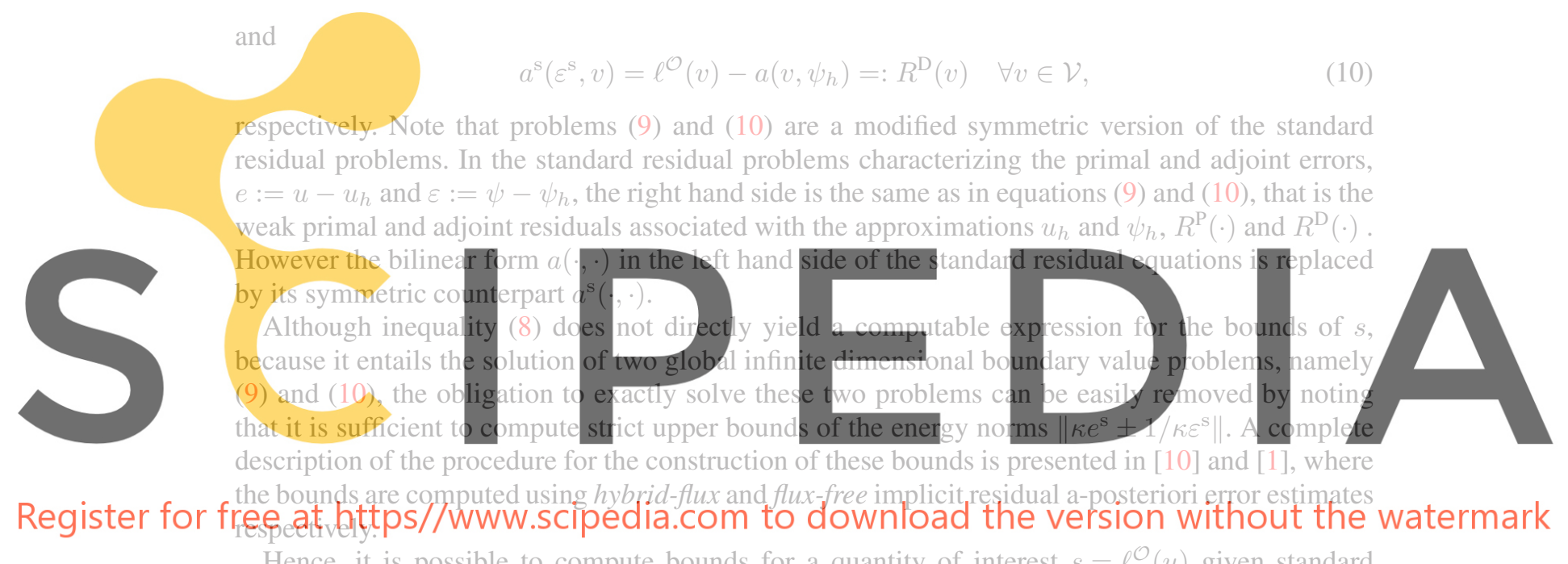

Hence, it is possible to compute bounds for a quantity of interest $s=\ell^{\mathcal{O}}(u)$ given standard Galerkin approximations of the primal and adjoint problems, $u_{h}$ and $\psi_{h}$. However, the techniques providing the bounds for $s$ are not directly applicable when the approximations $u_{h}$ and $\psi_{h}$ are computed using stabilized formulations.

The issues addressed in this article are: (i) can one obtain upper and lower bounds for the quantity of interest using stabilized approximations of the primal and adjoint problems, and if so, (ii) is it possible to extend the a posteriori error estimates given in [10] and [1] allowing to compute strict computable bounds?

The main difficulty of adapting the existing techniques to the use of stabilized methods is caused by the fact that in this case the weak primal and adjoint residuals fail to verify the standard orthogonality condition $-R^{\mathrm{P}}(v)$ and $R^{\mathrm{D}}(v)$ are not necessarily zero for $v \in \mathcal{V}^{h},-$ which is required both to derive inequality (8) and to formulate the residual type estimation strategies using a domain decomposition technique. Fortunately, a simple workaround allows to overcome this problem by introducing two straightforward modifications of the standard procedures. First, a similar expression to (8) holds by introducing some additional terms accounting for the non-orthogonality of the primal residual with respect to the finite element space $\mathcal{V}^{h}$. Second, the error estimation strategies yielding strict upper bounds for $\left\|\kappa e^{\mathrm{s}} \pm 1 / \kappa \varepsilon^{\mathrm{s}}\right\|^{2}$ are modified to handle error equations where the residuals, r.h.s of equations (9) and (10), do not verify the Galerkin orthogonality property.

The following result shows how inequality (8) is modified to account for the non-orthogonality of the residuals. The proof of this result is omitted here since it is analogous to the proof of theorem 1 in [1] - the only difference being that the term $a\left(e, \psi_{h}\right)=R^{\mathrm{P}}\left(\psi_{h}\right)$ appearing in the bounds does not necessarily vanish when working with stabilized approximations for the primal problem. 


\section{Theorem 1}

Let $e^{\mathrm{s}}$ and $\varepsilon^{\mathrm{s}} \in \mathcal{V}$ be such that for any $v \in \mathcal{V}$

$$
a^{\mathrm{s}}\left(e^{\mathrm{s}}, v\right)=R^{\mathrm{P}}(v) \quad \text { and } \quad a^{\mathrm{s}}\left(\varepsilon^{\mathrm{s}}, v\right)=R^{\mathrm{D}}(v) .
$$

Then,

$$
\ell^{\mathcal{O}}\left(u_{h}\right)+R^{\mathrm{P}}\left(\psi_{h}\right)-\frac{1}{4}\left\|\kappa e^{\mathrm{s}}-\frac{1}{\kappa} \varepsilon^{\mathrm{s}}\right\|^{2} \leq \ell^{\mathcal{O}}(u) \leq \ell^{\mathcal{O}}\left(u_{h}\right)+R^{\mathrm{P}}\left(\psi_{h}\right)+\frac{1}{4}\left\|\kappa e^{\mathrm{s}}+\frac{1}{\kappa} \varepsilon^{\mathrm{s}}\right\|^{2},
$$

and therefore

$$
\ell^{\mathcal{O}}\left(u_{h}\right)+R^{\mathrm{P}}\left(\psi_{h}\right)-\frac{1}{4}\left\|\kappa e^{\mathrm{s}}-\frac{1}{\kappa} \varepsilon^{\mathrm{s}}\right\|_{u b}^{2} \leq \ell^{\mathcal{O}}(u) \leq \ell^{\mathcal{O}}\left(u_{h}\right)+R^{\mathrm{P}}\left(\psi_{h}\right)+\frac{1}{4}\left\|\kappa e^{\mathrm{s}}+\frac{1}{\kappa} \varepsilon^{\mathrm{s}}\right\|_{u b}^{2},
$$

where $\|v\|_{u b}$ represents an upper bound for the value $\|v\|$.

Note that the above theorem is valid even if the approximations $u_{h}$ and $\psi_{h}$ are not computed using the SUPG finite element method, since no assumptions are made on these approximations. The above theorem is, then, a generalization of the bounding inequality (8) used to obtain bounds for outputs from Galerkin approximations of the primal and adjoint problems, where no requirements on $u_{h}$ and $\psi_{h}$ are done.

The importance of the above theorem is that it reduces the problem of obtaining upper and lower bounds for $s$ to obtaining upper bounds for the energy norm of the symmetric errors in the direct and adjoint problem. Using this result, a procedure to obtain bounds for $s$ may be sketched as follows:
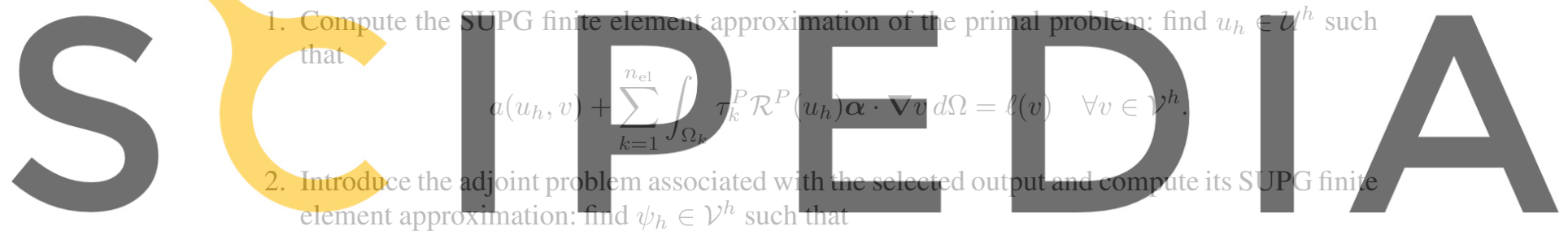

Register for free at https//www,scipedia..commoto downtoad the version,without the watermark

3. Recover the bounds for the output from the three following steps:

3.1 Introduce the modified symmetric versions of the residual problems: find $e^{\mathrm{s}}$ and $\varepsilon^{\mathrm{s}} \in \mathcal{V}$ such that

$$
a^{\mathrm{s}}\left(e^{\mathrm{s}}, v\right)=R^{\mathrm{P}}(v) \quad, \quad a^{\mathrm{s}}\left(\varepsilon^{\mathrm{s}}, v\right)=R^{\mathrm{D}}(v) \quad \forall v \in \mathcal{V},
$$

where $a^{\mathrm{S}}(\cdot, \cdot)$ is the symmetric counterpart of $a(\cdot, \cdot)$

$$
a^{\mathrm{s}}(w, v)=\int_{\Omega}[\nu \nabla w \cdot \nabla v+\tilde{\sigma} w v] \mathrm{d} \Omega+\frac{1}{2} \int_{\Gamma_{\mathrm{N}}} \boldsymbol{\alpha} \cdot \boldsymbol{n} w v \mathrm{~d} \Gamma .
$$

3.2 Compute the upper and lower bounds for $s, s^{l b} \leq s \leq s^{u b}$, as

$$
\begin{aligned}
s^{l b} & :=\ell^{\mathcal{O}}\left(u_{h}\right)+R^{\mathrm{P}}\left(\psi_{h}\right)-\frac{1}{4}\left\|\kappa e^{\mathrm{s}}-\frac{1}{\kappa} \varepsilon^{\mathrm{s}}\right\|_{u b}^{2} \\
s^{u b} & :=\ell^{\mathcal{O}}\left(u_{h}\right)+R^{\mathrm{P}}\left(\psi_{h}\right)+\frac{1}{4}\left\|\kappa e^{\mathrm{s}}+\frac{1}{\kappa} \varepsilon^{\mathrm{s}}\right\|_{u b}^{2},
\end{aligned}
$$

where $\|v\|_{u b}$ represents an upper bound for the value of $\|v\|$ and $\kappa \in \mathbb{R}$ is an arbitrary scalar non-zero parameter.

The computation of strict computable upper bounds for the energy norm forms the subject of next section. This approach is then used to compute $\left\|\kappa e^{\mathrm{s}} \pm 1 / \kappa \varepsilon^{\mathrm{s}}\right\|_{u b}^{2}$. 


\section{UPPER BOUNDS FOR THE ENERGY NORM: COMPLEMENTARY ENERGY RELAXATION}

Consider the auxiliary function $z \in \mathcal{V}$ solution of

$$
a^{\mathrm{s}}(z, v)=R^{*}(v) \quad \forall v \in \mathcal{V},
$$

where $R^{*}(v)=\alpha R^{\mathrm{P}}(v)+\beta R^{\mathrm{D}}(v)$ for $\alpha, \beta \in \mathbb{R}$. Note that $\alpha=1$ and $\beta=0$ yields $z=e^{\mathrm{s}}$ and that $\alpha=0$ and $\beta=1$ yields $z=\varepsilon^{\mathrm{s}}$. Moreover, $\alpha=\kappa$ and $\beta= \pm 1 / \kappa$ will be used later to obtain the required upper bounds for $\left\|\kappa e^{\mathrm{s}} \pm 1 / \kappa \varepsilon^{\mathrm{s}}\right\|^{2}$.

The purpose of this section is to establish a procedure to compute upper bounds of $\|z\|^{2}$. Note that the strategies presented in the series of papers $[10,3,4,18,5,1]$ may not be directly applied since they rely on the Galerkin orthogonality property of the residual $R^{*}(\cdot)$. In this work, two different approaches to recover upper bounds for $\|z\|^{2}$ are presented. The first approach is a modification of [1] which allows to recover bounds for $\|z\|^{2}$ from SUPG approximations of the primal and adjoint problems using a flux-free error estimation strategy. The second approach consists of taking some of the ideas presented in [10] and [19] to be able to recover strict bounds of $\|z\|^{2}$ using hybrid-flux strategies.

Both approaches rely on the use of the standard complementary energy approach. The key idea is to relax the continuous problem of finding $z \in \mathcal{V}$ fulfilling equation (14) into obtaining a pair of dual estimates $\hat{q} \in\left[\mathcal{L}^{2}(\Omega)\right]^{2}$ and $\hat{r} \in \mathcal{L}^{2}(\Omega)$ such that
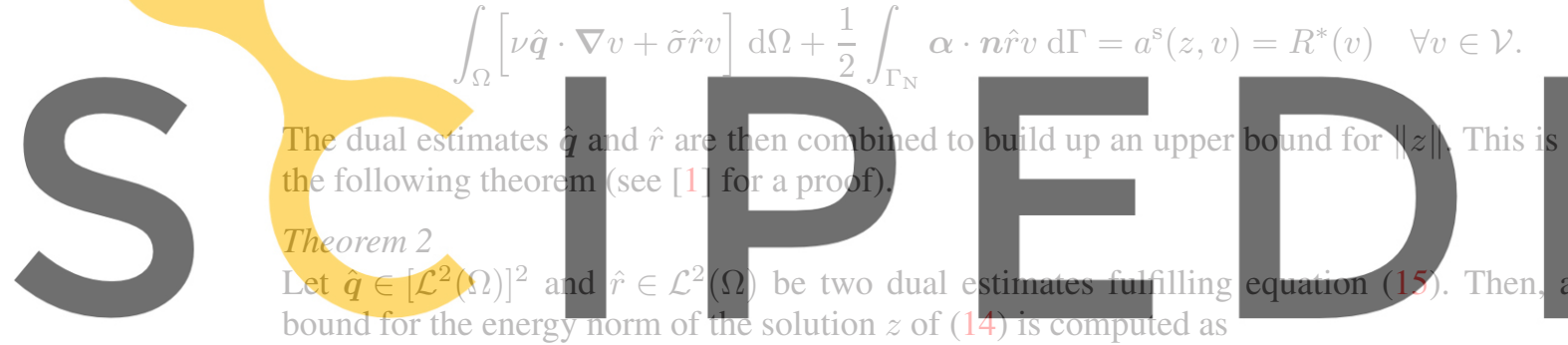

$(15)$

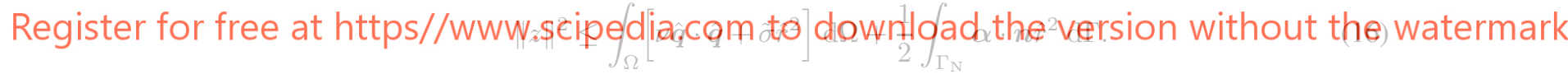

Moreover the previous inequality turns out to be an equality for $\hat{\boldsymbol{q}}=\nabla z$ and $\hat{r}=z$.

Theorem 2 allows to compute strict upper bounds for $\|z\|$ recovering two globally equilibrated dual estimates $\hat{\boldsymbol{q}}$ and $\hat{r}$, i.e. verifying equation (15). The essential feature of the method is that if the fields $f, g, f^{\mathcal{O}}$ and $g^{\mathcal{O}}$ are piecewise polynomial, it is possible to determine - amongst all the dual estimates $\hat{\boldsymbol{q}} \in\left[\mathcal{L}^{2}(\Omega)\right]^{2}$ and $\hat{r} \in \mathcal{L}^{2}(\Omega)$ verifying equation (15) - two piecewise polynomial fields verifying equation (15). That is, for a given suitable interpolation degree $q$, it is possible to find $\hat{\boldsymbol{q}} \in\left[\widehat{\mathbb{P}}^{q}(\Omega)\right]^{2}$ and $\hat{r} \in \widehat{\mathbb{P}}^{q}(\Omega)$ verifying equation (15) where

$$
\widehat{\mathbb{P}}^{q}(\Omega):=\left\{v \in \mathcal{L}^{2}(\Omega),\left.v\right|_{\Omega_{k}} \in \mathbb{P}^{q}\left(\Omega_{k}\right)\right\},
$$

see $[10,20]$. A more detailed discussion on the proper choice of the interpolation degree $q$ is given in sections 4.2 and 4.3 .

Therefore, the computation of strict upper bounds for $\|z\|$ is reduced to a discrete problem: determine $\hat{\boldsymbol{q}} \in\left[\widehat{\mathbb{P}}^{q}(\Omega)\right]^{2}$ and $\hat{r} \in \widehat{\mathbb{P}}^{q}(\Omega)$ verifying equation (15). Moreover, for a fixed $q \in \mathbb{N}$ the optimal choice is to determine $\hat{\boldsymbol{q}}$ and $\hat{r}$ verifying (15) and minimizing the upper bound

$$
\int_{\Omega}\left[\nu \hat{\boldsymbol{q}} \cdot \hat{\boldsymbol{q}}+\tilde{\sigma} \hat{r}^{2}\right] \mathrm{d} \Omega+\frac{1}{2} \int_{\Gamma_{\mathrm{N}}} \boldsymbol{\alpha} \cdot \boldsymbol{n} \hat{r}^{2} \mathrm{~d} \Gamma .
$$

This problem is discrete (with finite number of d.o.f.) but global, that is, affecting the whole domain $\Omega$. Thankfully, proper domain decomposition techniques allow decomposing the global discrete 
problem into local problems. That is, the piecewise polynomial fields $\hat{\boldsymbol{q}}$ and $\hat{r}$ are to be computed solving local discrete problems.

However, the existing domain decomposition techniques can not be directly applied if the residual $R^{*}(\cdot)$ does not verify the Galerkin orthogonality condition. This section considers the two most used classical domain decomposition techniques - the flux-free approach and the hybrid-flux approach and extends these techniques to be able to deal with non orthogonal residuals.

Recall that the flux-free is based on the partition-of-unity property which is used to localize the problems in $\Omega$ to subdomains different than elements. That is, the local problems for the dual estimates $\hat{\boldsymbol{q}}$ and $\hat{r}$ are posed over patches of elements. By contrast, in the hybrid-flux approach the dual estimates $\hat{\boldsymbol{q}}$ and $\hat{r}$ are computed solving local independent problems in each element of the mesh. This requires the use of flux-equilibration techniques to properly set the boundary conditions for the local elementary problems. First, the equilibrated residual method is used to compute the equilibrated fluxes at the interelementary edges of the mesh and these fluxes are then used as local boundary conditions to compute the dual estimates $\hat{\boldsymbol{q}}$ and $\hat{r}$ in each triangle of the mesh. The advantage of the flux-free approach is that the local problems are self-equilibrated and therefore it avoids the use of flux-equilibration techniques.

\subsection{Modified Galerkin orthogonality property}

Reeall that in the case that $u_{h}$ and $\psi_{h}$ are not computed using the standard Galerkin method, the residuals $R^{\mathrm{P}}(v)$ and $R^{\mathrm{D}}(v)$, and thus $R^{*}(v)$, do not verify the Galerkin orthogonality property, that is, also $R^{*}(v)$ is not necessarily zero for $v \in \mathcal{V}^{h}$.

However, from equations (3) and (7), the primal and adjoint residuals satisfy
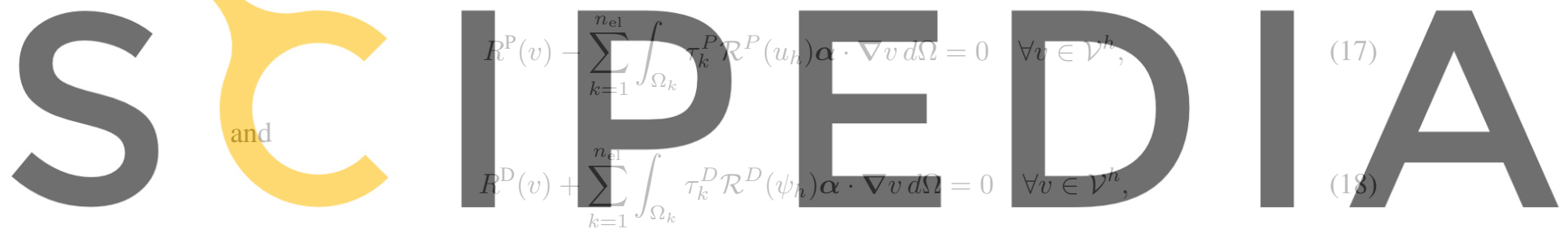

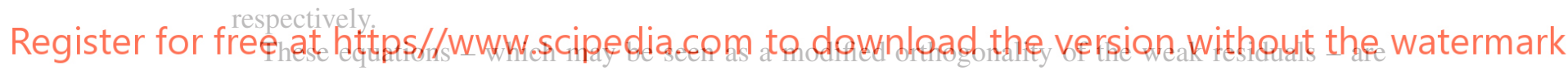
an essential tool to develop the error estimation strategies presented in this section. Henceforth, equations (17) and (18) will be named after modified orthogonality properties.

Nota that multiplying equations (17) and (18) by the coefficients $\alpha$ and $\beta$ respectively, yield the subsequent modified orthogonality of the combined residual $R^{*}(\cdot)$

$$
R^{*}(v)+\sum_{k=1}^{n_{\mathrm{el}}} \int_{\Omega_{k}}\left(-\alpha \tau_{k}^{P} \mathcal{R}^{P}\left(u_{h}\right)+\beta \tau_{k}^{D} \mathcal{R}^{D}\left(\psi_{h}\right)\right) \boldsymbol{\alpha} \cdot \nabla v d \Omega=0 \quad \forall v \in \mathcal{V}^{h}
$$

\subsection{Local computation of the dual estimates $\hat{\boldsymbol{q}}$ and $\hat{r}$ using a flux-free approach}

This section is devoted to detail the computation of the piecewise polynomial dual estimates $\hat{\boldsymbol{q}}$ and $\hat{r}$ using the flux-free approach proposed in [11]. The strategy proposed in [1] can not be directly applied since the residuals are not orthogonal to $\mathcal{V}^{h}$. However, a simple workaround is proposed, using the modified orthogonality properties of the primal and adjoint residuals, equations (17), (18) and (19).

Let $\boldsymbol{x}^{i} i=1, \ldots, n_{\mathrm{np}}$ denote the vertices of the elements (triangles) in the computational mesh (thus linked to $\mathcal{U}^{h}$ ) and $\phi^{i}$ denote the corresponding linear shape functions, which are such that $\phi^{i}\left(\boldsymbol{x}^{j}\right)=\delta_{i j}$. The support of $\phi^{i}$ is denoted by $\omega^{i}$ and it is called the star centered in, or associated with, vertex $\boldsymbol{x}^{i}$. It is important to recall that the linear shape functions based on the vertices are a partition of unity, namely

$$
\sum_{i=1}^{n_{\mathrm{np}}} \phi^{i}=1
$$


Let also $\mathcal{V}\left(\omega^{i}\right)$ and $\widehat{\mathbb{P}^{q}}\left(\omega^{i}\right)$ denote the local restrictions of the spaces $\mathcal{V}$ and $\widehat{\mathbb{P}^{q}}(\Omega)$ to the star $\omega^{i}$. Formally any function $v \in \mathcal{V}\left(\omega^{i}\right)$ or $v \in \widehat{\mathbb{P}}^{q}\left(\omega^{i}\right)$ is not defined in the whole domain $\Omega$ but only in the star $\omega^{i}$. However, here any $v \in \mathcal{V}\left(\omega^{i}\right)$ or $v \in \widehat{\mathbb{P}}^{q}\left(\omega^{i}\right)$ is naturally extended to $\Omega$ by setting the values outside $\omega^{i}$ to zero. Thus, functions in $\mathcal{V}\left(\omega^{i}\right)$ are $\mathcal{H}^{1}$ functions in $\omega^{i}$ but generally discontinuous across the boundary of the star $\omega^{i}$, whereas functions in $\widehat{\mathbb{P}} q\left(\omega^{i}\right)$ are piecewise polynomial functions in the triangles contained in $\omega^{i}$ vanishing on the elements outside $\omega^{i}$.

The dual estimates $\hat{\boldsymbol{q}}$ and $\hat{r}$ are computed as

$$
\hat{\boldsymbol{q}}=\sum_{i=1}^{n_{\mathrm{np}}} \hat{\boldsymbol{q}}^{i} \quad \text { and } \quad \hat{r}=\sum_{i=1}^{n_{\mathrm{np}}} \hat{r}^{i}
$$

where the local estimates $\hat{\boldsymbol{q}}^{i} \in\left[\widehat{\mathbb{P}}^{q}\left(\omega^{i}\right)\right]^{2}$ and $\hat{r}^{i} \in \widehat{\mathbb{P}}^{q}\left(\omega^{i}\right)$, defined inside the star $\omega^{i}$, are such that for any $v \in \mathcal{V}\left(\omega^{i}\right)$

$$
\begin{aligned}
& \int_{\omega^{2}}\left[\nu \hat{q}^{i} \cdot \nabla v+\tilde{\sigma} \hat{r}^{i} v\right] \mathrm{d} \Omega+\frac{1}{2} \int_{\Gamma_{\mathrm{N}} \cap \partial \omega^{i}} \alpha \cdot n \hat{r}^{i} v \mathrm{~d} \Gamma=R^{*}\left(\phi^{i} v\right)+\sum_{\Omega_{k} \subset \omega^{i}} \int_{\Omega_{k}} f_{i}^{\perp} v d \Omega \\
& \qquad f_{i}^{\perp}=\left(-\alpha \tau_{k}^{P} \mathcal{R}^{P}\left(u_{h}\right)+\beta \tau_{k}^{D} \mathcal{R}^{D}\left(\psi_{h}\right)\right) \alpha \cdot \nabla \phi^{i} .
\end{aligned}
$$

where

\section{Remark 2}

Note that in [1], the r.h.s. of the local problems for $\hat{q}^{i}$ and $\hat{r}^{i}$ is simply $R^{*}\left(\phi^{i} v\right)$. If the same r.h.s. is chosen here, the local problen (22) is no necess arily solvable, that is, it does not necessarily admit a solution. The nev while preserving the gl

This new definition solution. Indeed, i intersects the Neumann boundary and $\left.\alpha \cdot n\right|_{\Gamma-1}$

is ensured. On the contrary, the kernel of the bilinear operator appearing in the 1.h.s. is the one dimensional space of constants, $\mathbb{P}^{0}\left(\omega^{i}\right)$, and equation (22) is solyable if and only if the compatibility

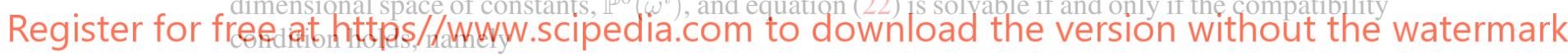

$$
R^{*}\left(\phi^{i} c\right)+\sum_{\Omega_{k} \subset \omega^{i}} \int_{\Omega_{k}} f_{i}^{\perp} c d \Omega=0 \quad \forall c \in \mathbb{P}^{0}\left(\omega^{i}\right)
$$

Now, substituting the definition of $f_{i}^{\perp}$ into the previous equation, taking into account that $c$ is constant in the star $\omega^{i}$ and finally noting that the support of the function $\nabla \phi^{i}$ is the star $\omega^{i}$, yields that the compatibility condition is equivalent to

$$
\begin{aligned}
0 & =c R^{*}\left(\phi^{i}\right)+\sum_{\Omega_{k} \subset \omega^{i}} \int_{\Omega_{k}}\left(-\alpha \tau_{k}^{P} \mathcal{R}^{P}\left(u_{h}\right)+\beta \tau_{k}^{D} \mathcal{R}^{D}\left(\psi_{h}\right)\right) \boldsymbol{\alpha} \cdot \boldsymbol{\nabla} \phi^{i} c d \Omega \\
& =c\left[R^{*}\left(\phi^{i}\right)+\sum_{k=1}^{n_{\mathrm{el}}} \int_{\Omega_{k}}\left(-\alpha \tau_{k}^{P} \mathcal{R}^{P}\left(u_{h}\right)+\beta \tau_{k}^{D} \mathcal{R}^{D}\left(\psi_{h}\right)\right) \boldsymbol{\alpha} \cdot \boldsymbol{\nabla} \phi^{i} d \Omega\right]
\end{aligned}
$$

which follows replacing $v=\phi^{i} \in \mathcal{V}^{h}$ in equation (19).

Theorem 3

The dual estimates $\hat{\boldsymbol{q}}=\sum_{i=1}^{n_{\mathrm{np}}} \hat{\boldsymbol{q}}^{i}$ and $\hat{r}=\sum_{i=1}^{n_{\mathrm{np}}} \hat{r}^{i}$, where $\hat{\boldsymbol{q}}^{i}$ and $\hat{r}^{i}$ verify the local problems given in (22), verify the hypothesis of theorem 2 and therefore

$$
\|z\|^{2} \leq \int_{\Omega}\left[\nu \hat{\boldsymbol{q}} \cdot \hat{\boldsymbol{q}}+\tilde{\sigma} \hat{r}^{2}\right] \mathrm{d} \Omega+\frac{1}{2} \int_{\Gamma_{\mathrm{N}}} \boldsymbol{\alpha} \cdot \boldsymbol{n} \hat{r}^{2} \mathrm{~d} \Gamma .
$$




\section{Proof}

The dual estimates $\hat{\boldsymbol{q}}$ and $\hat{r}$ verify equation (15) and therefore theorem 3 is a straightforward particularization of theorem 2. Indeed, let $v \in \mathcal{V}$ which implies $\left.v\right|_{\omega^{i}} \in \mathcal{V}\left(\omega^{i}\right)$ and consider the definition of the dual estimates - equation (21) — and the local equations (22) to obtain

$$
\begin{aligned}
\int_{\Omega}[\nu \hat{\boldsymbol{q}} \cdot \boldsymbol{\nabla} & v+\tilde{\sigma} \hat{r} v] \mathrm{d} \Omega+\frac{1}{2} \int_{\Gamma_{\mathrm{N}}} \boldsymbol{\alpha} \cdot \boldsymbol{n} \hat{r} v \mathrm{~d} \Gamma \\
& =\sum_{i=1}^{n_{\mathrm{np}}}\left\{\int_{\omega^{i}}\left[\nu \hat{\boldsymbol{q}}^{i} \cdot \nabla v+\tilde{\sigma} \hat{r}^{i} v\right] \mathrm{d} \Omega+\frac{1}{2} \int_{\Gamma_{\mathrm{N}} \cap \partial \omega^{i}} \boldsymbol{\alpha} \cdot \boldsymbol{n} \hat{r}^{i} v \mathrm{~d} \Gamma\right\} \\
& =\sum_{i=1}^{n_{\mathrm{np}}}\left\{R^{*}\left(\phi^{i} v\right)+\sum_{\Omega_{k} \subset \omega^{i}} \int_{\Omega_{k}} f_{i}^{\perp} v d \Omega\right\} .
\end{aligned}
$$

Then, rearranging terms using the linearity of the residual $R^{*}(\cdot)$, the partition-of-unity property equation (20) - and

$$
\sum_{\omega^{i} \cap \Omega_{k} \neq \emptyset} f_{i}^{\perp}=0
$$

\section{yields the desired result}
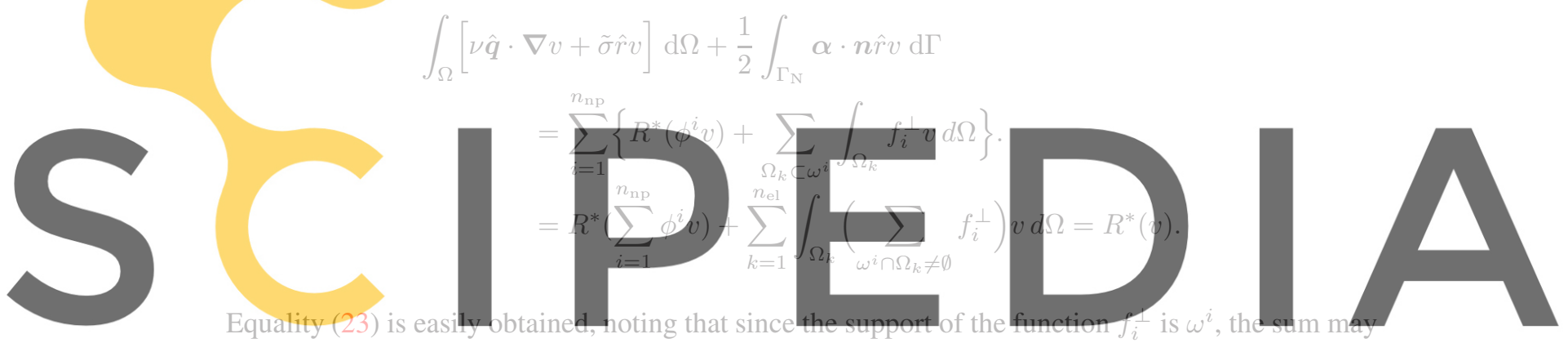

be extended not only to the stars intersecting $\Omega_{k}$ but to all the stars, and then rearranging terms:

Register for free at https//www.scipedia.com to download the version without the watermark

$$
\begin{aligned}
\sum_{\omega_{i} \cap \Omega_{k} \neq \emptyset} f_{i}^{\perp} & =\sum_{i=1} f_{i}^{\perp}=\sum_{i=1}\left(-\alpha \tau_{k}^{P} \mathcal{R}^{P}\left(u_{h}\right)+\beta \tau_{k}^{D} \mathcal{R}^{D}\left(\psi_{h}\right)\right) \alpha \cdot \nabla \phi^{i} \\
& =\left(-\alpha \tau_{k}^{P} \mathcal{R}^{P}\left(u_{h}\right)+\beta \tau_{k}^{D} \mathcal{R}^{D}\left(\psi_{h}\right)\right) \boldsymbol{\alpha} \cdot \boldsymbol{\nabla}\left(\sum_{i=1}^{n_{\mathrm{np}}} \phi^{i}\right) \\
& =\left(-\alpha \tau_{k}^{P} \mathcal{R}^{P}\left(u_{h}\right)+\beta \tau_{k}^{D} \mathcal{R}^{D}\left(\psi_{h}\right)\right) \boldsymbol{\alpha} \cdot \boldsymbol{\nabla} 1=0
\end{aligned}
$$

where the partition-of-unity property — equation (20) — has been used.

The computation of the dual estimates $\hat{\boldsymbol{q}}^{i}$ and $\hat{r}^{i}$ verifying equation (22) is done using the same strategy as in [1]. Note that the only difference between the computation of the estimates when introducing stabilization techniques is the new term accounting for the non-orthogonality of the residuals appearing in the local equations. This new added term

$$
\sum_{\Omega_{k} \subset \omega^{i}} \int_{\Omega_{k}} f_{i}^{\perp} v d \Omega,
$$

which vanishes if no stabilization is used, $\tau_{k}^{P}=\tau_{k}^{D}=0$, involves only a modification of the source term of the local problem.

Thus, following the notation used in [1], the r.h.s. of equation (22) can be rewritten as

$$
R^{*}\left(\phi_{i} v\right)=\int_{\omega^{i}} f_{i}^{*} v \mathrm{~d} \Omega+\int_{\Gamma_{N} \cap \partial \omega^{i}} g_{i}^{*} v \mathrm{~d} \Gamma-\int_{\omega^{i}} \nu \hat{\boldsymbol{q}}_{h}^{i} \cdot \nabla v \mathrm{~d} \Omega
$$


where the following compact notation is introduced

$$
\begin{aligned}
& f_{i}^{*}=\alpha\left[\phi_{i} f-\phi_{i} \boldsymbol{\alpha} \cdot \boldsymbol{\nabla} u_{h}-\sigma \phi_{i} u_{h}-\nu \boldsymbol{\nabla} u_{h} \cdot \boldsymbol{\nabla} \phi_{i}\right] \\
& +\beta\left[\phi_{i} f^{\mathcal{O}}-\psi_{h} \boldsymbol{\alpha} \cdot \boldsymbol{\nabla} \phi_{i}-\sigma \phi_{i} \psi_{h}-\nu \boldsymbol{\nabla} \psi_{h} \cdot \boldsymbol{\nabla} \phi_{i}\right]+f_{i}^{\perp}, \\
& g_{i}^{*}=\alpha \phi_{i} g+\beta \phi_{i} g^{\mathcal{O}} \quad \text { and } \quad \hat{\boldsymbol{q}}_{h}^{i}=\alpha \phi_{i} \boldsymbol{\nabla} u_{h}+\beta\left(\phi_{i} \boldsymbol{\nabla} \psi_{h}+\frac{1}{\nu} \phi_{i} \psi_{h} \boldsymbol{\alpha}\right),
\end{aligned}
$$

and therefore, introducing the new unknown $\hat{\boldsymbol{q}}_{*}^{\perp i}=\hat{\boldsymbol{q}}^{i}+\hat{\boldsymbol{q}}_{h}^{i}$, the strong form to compute the dual estimates $\hat{\boldsymbol{q}}_{*}^{\perp i} \in\left[\widehat{\mathbb{P}}^{q}\left(\omega^{i}\right)\right]^{2}$ and $\hat{r}^{i} \in \widehat{\mathbb{P}}^{q}\left(\omega^{i}\right)$ is,

$$
\begin{array}{cl}
-\nu \nabla \cdot \hat{\boldsymbol{q}}_{*}^{\perp i}+\tilde{\sigma} \hat{r}^{i}=f_{i}^{*} & \text { in } \omega^{i} \\
\nu \hat{\boldsymbol{q}}_{*}^{\perp i} \cdot \boldsymbol{n}+\frac{1}{2} \boldsymbol{\alpha} \cdot \boldsymbol{n} \hat{r}^{i}=g_{i}^{*} & \text { on } \gamma \in \Gamma_{\mathrm{N}} \cap \partial \omega^{i} \\
\nu \hat{q}_{*}^{\perp i} \cdot n=0 & \text { on } \gamma \in \partial \omega^{i}-\left\{\Gamma_{\mathrm{N}} \cup \Gamma_{\mathrm{D}}\right\} \\
\left.\nu \hat{q}_{*}^{\perp i}\right|_{\Omega_{k}} \cdot n_{k}+\left.\nu \hat{q}_{*}^{\perp i}\right|_{\Omega_{l}} \cdot n_{l}=0 & \text { on } \gamma \in \partial \Omega_{k} \cap \partial \Omega_{l}, \quad \Omega_{k}, \Omega_{l} \subset \omega^{i},
\end{array}
$$

where $n_{k}$ and $n_{l}$ are the outward normal to the elements $\Omega_{k}$ and $\Omega_{l}$ respectively. See [1] for a detailed derivation of the strong form of the local problem (22).

Remark 3

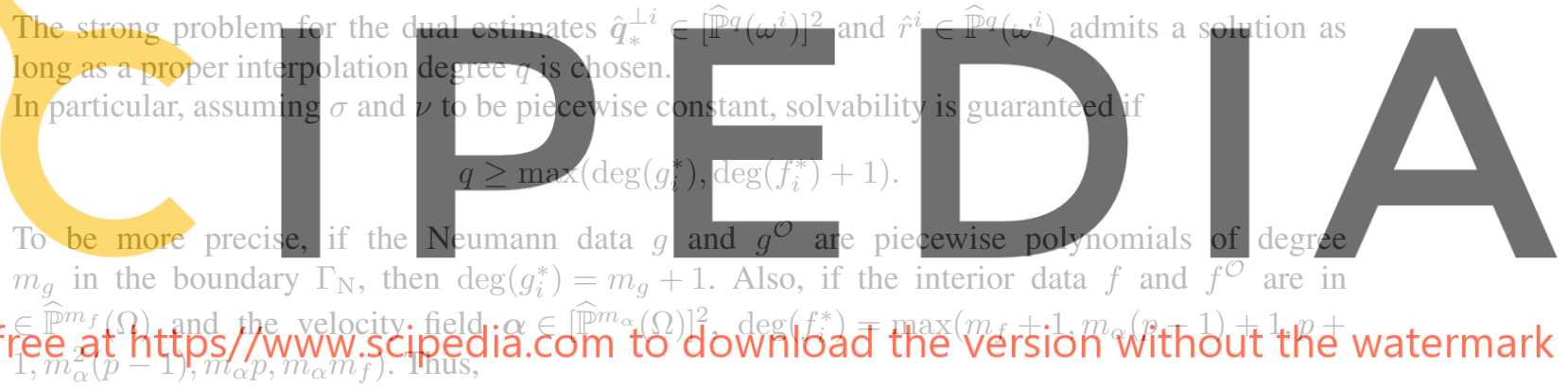

$$
q \geq \max \left(m_{g}+1, m_{f}+2, m_{\alpha} p+1, p+2, m_{\alpha}^{2}(p-1)+1, m_{\alpha} m_{f}+1\right),
$$

The previous restriction is the worst case scenario, since depending on the problem to be solved, for instance for problems without reaction term $\sigma=0$ or without applied Neumann boundary conditions (or homogeneous ones), some of the restrictions can be removed or weakened. In particular, in advection-diffusion problems associated to $\sigma=0$, the term $q \geq p+2$ may be replaced by $q \geq p+1$. Also, it is worth noting that the last two terms in equation (24), namely $m_{\alpha}^{2}(p-1)+1$ and $m_{\alpha} m_{f}+1$, only appear when stabilization techniques are used. Even in this case, for piecewise constant or linear velocity fields, these terms have no influence in the selection of the interpolation degree $q$.

\subsection{Local computation of the dual estimates $\hat{\boldsymbol{q}}$ and $\hat{r}$ using hybrid-flux techniques}

This section is devoted to detail the computation of the piecewise polynomial dual estimates $\hat{\boldsymbol{q}}$ and $\hat{r}$ using the hybrid-flux technique described in [13]. In fact this strategy is a modification of the technique presented in [10] - which provides a tool to compute strict bounds for quantities of interest for the advection-reaction-diffusion equation using standard Galerkin approximations of the primal and adjoint problems - based on the strategy developed in [19] - which provides a tool to compute asymptotic bounds for quantities of interest from SUPG method approximations of the primal and adjoint problems.

Hybrid-flux methods (or equilibrated residual methods) may be seen as a domain decomposition strategy which allows to decompose the global problem (15) into solving local problems in each 
element of the finite element mesh. This approach is standard and it is widely used in a posteriori error estimation for steady problems $[13,21,14]$. The key point is to be able to compute equilibrated fluxes at the interelementary edges of the mesh which are then used as local boundary conditions for the local elementary problems. Standard constructions of the equilibrated fluxes require the r.h.s. of the residual problem given in (15), that is, $R^{*}(\cdot)$, to be orthogonal to the finite element space $\mathcal{V}^{h}$. However, the strategy proposed in [19], may be used in the context of the SUPG method to provide a simple workaround to the problem of $R^{*}(\cdot)$ being non-orthogonal to $\mathcal{V}^{h}$.

Equilibrated residual methods compute the dual fields $\hat{\boldsymbol{q}}$ and $\hat{r}$ verifying equation (15), by means of computing two piecewise polynomial fields $\hat{\boldsymbol{q}} \in\left[\widehat{\mathbb{P}}^{q}(\Omega)\right]^{2}$ and $\hat{r} \in \widehat{\mathbb{P}}^{q}(\Omega)$ such that

$$
\int_{\Omega}[\nu \hat{\boldsymbol{q}} \cdot \nabla v+\tilde{\sigma} \hat{r} v] \mathrm{d} \Omega+\frac{1}{2} \int_{\Gamma_{\mathrm{N}}} \boldsymbol{\alpha} \cdot \boldsymbol{n} \hat{r} v \mathrm{~d} \Gamma=R^{*}(v)+\sum_{\gamma \in \Gamma_{h}} \int_{\gamma} \lambda[v] \mathrm{d} \Gamma \quad \forall v \in \widehat{\mathcal{V}} .
$$

Here, the "broken" space $\widehat{\mathcal{V}}$ is $\widehat{\mathcal{V}}:=\left\{v \in \mathcal{L}^{2}(\Omega),\left.v\right|_{\Omega_{k}} \in \mathcal{H}^{1}\left(\Omega_{k}\right)\right\}$, that is, functions in $\widehat{\mathcal{V}}$ are allowed to present discontinuities across the edges of the mesh and are not forced to verify the Dirichlet boundary conditions, $\Gamma_{h}$ denotes the set of all the edges contained in the interior of the mesh or on the Dirichlet boundary, $\lambda \in \prod_{k=1}^{n_{\text {el }}} \mathcal{H}^{-\frac{1}{2}}\left(\partial \Omega_{k}\right)$ are the equilibrated fluxes added to the r.h.s. of equation (25) in order to yield equilibrated and thus solvable local problems in each element and $\left.[v]\right|_{\gamma}$ is the jump of the function $v$ along the edge $\gamma$ if it is an interior edge or $\left.[v]\right|_{\partial \Omega}=v$ for the exterior edges. In order to properly define the jump of a function across the mesh edges, an arbitrary ordering of the elements of the mesh is introduced and $\varsigma_{k}$ is defined as

$$
\varsigma_{k}(\boldsymbol{x})= \begin{cases}-1 & \boldsymbol{x} \in \bar{\Omega}_{k} \cap \bar{\Omega}_{l}, k<l \\ +1 & \text { otherwise }\end{cases}
$$

In this case

$$
\left.[v]\right|_{\gamma}= \begin{cases}\left.v\right|_{\Omega_{k}} \varsigma_{k}+\left.v\right|_{\Omega_{l}} \varsigma_{l} & \text { if } \gamma=\Omega_{k} \cap \Omega_{l} \in \Gamma_{h} \\ v & \text { if } \gamma \in \Gamma_{\mathrm{D}},\end{cases}
$$

where the values of $\left.v\right|_{\Omega_{k}}$ and $\left.v\right|_{\Omega_{l}}$ at the edge $\gamma$ are computed in using the traces of the funcions $\left.v\right|_{\Omega_{k}}$ and $\left.v\right|_{\Omega_{l}}$ on $\gamma$.

The different existing equilibration techniques differ in the choice of the equilibrated fluxes $\lambda$ which may be computed with an asymptotic complexity that is linear in the number of vertices of the mesh using, for instance, the procedure proposed by Ladevèze and Leguillon in [13].

It is a relatively simple matter to see that the dual estimates $\hat{\boldsymbol{q}}$ and $\hat{r}$ computed from equation (25) verify equation (15). Indeed, for any $v \in \mathcal{V}$, that is, for any $v$ in $\mathcal{H}^{1}(\Omega)$ vanishing on the Dirichlet boundary of the domain,

$$
\int_{\gamma} \lambda[v] \mathrm{d} \Gamma=0
$$

for all $\gamma \in \Gamma_{h}, \lambda \in \prod_{k=1}^{n_{\text {el }}} \mathcal{H}^{-\frac{1}{2}}\left(\partial \Omega_{k}\right)$. Therefore, taking $v \in \mathcal{V} \subset \widehat{\mathcal{V}}$ in equation (25) yields

$$
\int_{\Omega}[\nu \hat{\boldsymbol{q}} \cdot \boldsymbol{\nabla} v+\tilde{\sigma} \hat{r} v] \mathrm{d} \Omega+\frac{1}{2} \int_{\Gamma_{\mathrm{N}}} \boldsymbol{\alpha} \cdot \boldsymbol{n} \hat{r} v \mathrm{~d} \Gamma=R^{*}(v)+\sum_{\gamma \in \Gamma_{h}} \int_{\gamma} \lambda[v] \mathrm{d} \Gamma=R^{*}(v),
$$

as required in equation (15).

Note that for a given choice of the equilibrated fluxes $\lambda$, the dual estimates $\hat{\boldsymbol{q}}$ and $\hat{r}$ solution of (25) can be computed solving independent problems posed over the elements of the mesh: find $\hat{\boldsymbol{q}}^{k} \in\left[\mathbb{P}^{q}\left(\Omega_{k}\right)\right]^{2}$ and $\hat{r}^{k} \in \mathbb{P}^{q}\left(\Omega_{k}\right)$ such that

$$
\int_{\Omega_{k}}\left[\nu \hat{\boldsymbol{q}}^{k} \cdot \nabla v+\tilde{\sigma} \hat{r}^{k} v\right] \mathrm{d} \Omega+\frac{1}{2} \int_{\Gamma_{\mathrm{N}} \cap \partial \Omega_{k}} \boldsymbol{\alpha} \cdot \boldsymbol{n} \hat{r}^{k} v \mathrm{~d} \Gamma=R_{k}^{*}(v)+\int_{\partial \Omega_{k} \backslash \Gamma_{\mathrm{N}}} \varsigma_{k} \lambda v \mathrm{~d} \Gamma \quad \forall v \in \mathcal{H}^{1}\left(\Omega_{k}\right) .
$$




\section{Remark 4}

It is tacitly assumed that problems given in equation (26) have at least one solution. For elements $\Omega_{k}$ associated with a strictly positive reaction term $\left.\tilde{\sigma}\right|_{\Omega_{k}}>0$ or intersecting the Neumann boundary and $\left.\boldsymbol{\alpha} \cdot \boldsymbol{n}\right|_{\Gamma_{\mathrm{N}} \cap \partial \Omega_{k}} \neq 0$ the kernel of the r.h.s. of equation (26) is empty, and therefore, equation (26) has a unique solution. On the contrary, the kernel of the r.h.s. are the constant functions. In this case, the problem is solvable if and only if the following compatibility condition holds:

$$
R_{k}^{*}(1)+\int_{\partial \Omega_{k} \backslash \Gamma_{\mathrm{N}}} \varsigma_{k} \lambda \mathrm{d} \Gamma=0,
$$

that is, if the r.h.s. of equation (26) vanishes for $v=\left.1\right|_{\Omega_{k}}$. This previous condition expresses that the boundary data must be in equilibrium with the interior load so that the local problems are solvable. This is precisely the required condition for the fluxes $\lambda$ to be equilibrated.

\section{Remark 5}

In order to enforce the compatibility condition, equation (27), the equilibrated fluxes $\lambda$, in the case where $u_{h}$ and $\psi_{h}$ are the Galerkin approximations of $u$ and $\psi$, are forced to verify

$$
R^{*}(v)+\sum_{\gamma \in \Gamma_{h}} \int_{\gamma} \lambda[v] \mathrm{d} \Gamma=0 \quad \forall v \in \widehat{\mathcal{V}}^{h},
$$

where $\widehat{\mathcal{V}}^{h}$ is obtained from $\mathcal{V}^{h}$ relaxing both the Dirichlet homogeneous boundary conditions and the continuity of the functions across the edges of $\Gamma_{h}$. Note that for $\left.1\right|_{\Omega_{k}} \in \widehat{\mathcal{V}}^{h}$, the previous condition yields to the compatibility condition. However, when using the SUPG approximations, it is not possible to compute a set of equilibrated fluxes $\lambda$ verifying equation (28) due to the non-orthogonality of the residual $R^{*}(\cdot)$ with respect to $\mathcal{V}^{h}$. Indeed, take $v \in \mathcal{V} \subset \widehat{\mathcal{V}}^{h}$, then since $\left.[v]\right|_{\gamma}=0 \forall \gamma \in \Gamma_{h}$ equation (28) becomes $R^{*}(v)=0$ which does not necessarily hold.

Luckily, [19] proposes a simple workaround to this problem. The equilibrated fluxes are forced to verify

$$
R^{*}(v)+\sum_{k=1}^{n_{\mathrm{el}}} \int_{\Omega_{k}}\left(-\alpha \tau_{k}^{P} \mathcal{R}^{P}\left(u_{h}\right)+\beta \tau_{k}^{D} \mathcal{R}^{D}\left(\psi_{h}\right)\right) \boldsymbol{\alpha} \cdot \nabla v d \Omega+\sum_{\gamma \in \Gamma_{h}} \int_{\gamma} \lambda[v] \mathrm{d} \Gamma=0 \quad \forall v \in \widehat{\mathcal{V}}^{h},
$$

instead of equation (28). Note that again for $\left.1\right|_{\Omega_{k}} \in \widehat{\mathcal{V}}^{h}$, the previous condition yields to the compatibility condition, since the additional term vanishes for $v$ being constant inside the elements of the mesh. Moreover, the set of conditions posed by equations (29) are now compatible since for any $v \in \mathcal{V}^{h} \subset \widehat{\mathcal{V}}^{h}$,

$$
R^{*}(v)+\sum_{k=1}^{n_{\mathrm{el}}} \int_{\Omega_{k}}\left(-\alpha \tau_{k}^{P} \mathcal{R}^{P}\left(u_{h}\right)+\beta \tau_{k}^{D} \mathcal{R}^{D}\left(\psi_{h}\right)\right) \boldsymbol{\alpha} \cdot \nabla v d \Omega=0,
$$

due to equation (19).

Therefore, the strategy to compute the dual estimates $\hat{\boldsymbol{q}}$ and $\hat{r}$ solution of (25), is equivalent to the strategy proposed in [10], that is, for each element of the mesh, the restriction of the dual estimates $\hat{\boldsymbol{q}}$ and $\hat{r}$ to the element, $\hat{\boldsymbol{q}}^{k}$ and $\hat{r}^{k}$, are computed solving the local equation (26). The only difference is that now, the equilibrated fluxes are found solving the modified equation (29).

\section{Remark 6}

The strong problem for the dual estimates (26) admits a solution as long as a proper interpolation degree $q$ is chosen, see [10]. The same derivation applies in this context since the stabilization term only affects to the computation of the equilibrated fluxes which again can be taken to be functions of degree $p$ in the edges of the mesh independently of the stabilization terms. In particular, following the notation of remark 3 it can be stated that

$$
q \geq \max \left(m_{g}, m_{f}+1, m_{\alpha} p, p+1\right),
$$


As in the flux-free context, the previous restriction is the worst case scenario. In particular, the term $q \geq p+1$ appears only for $\sigma>0$. For $\sigma=0$ this restriction turns into $q \geq p$.

Thus, regarding the choice of the interpolation degree of the dual estimates, $q$, the hybrid-flux technique presents two advantages: 1) the local problems are not weighted by the linear shape functions $\phi^{i}$ and therefore the minimum value of the local polynomial order, $q$, is one less than for the flux-free technique, and 2) the stabilization term plays a role only in computing the equilibrated fluxes, $\lambda$. Thus, the choice of the interpolation degree $q$ depends linearly on $m_{\alpha}$ (and not quadratically as in the flux-free case for stabilized techniques).

\subsection{Computational cost versus accuracy}

This section is devoted to compare the computational effort required to solve the local problems for both the flux-free and the hybrid-flux approach versus the accuracy of the methods. The explanation given herein, is valid whether the bounds are computed either using stabilized or standard finite element techniques, since the presented extension does not affect the computational cost of the methods. However, this section is included to clarify and illustrate the resemblances/differences of the two presented strategies.

In both cases, the cost of computing strict upper bounds for quantities of interest is proportional to the number of vertex nodes in the mesh once the adjoint finite element approximation has been computed. Indeed, given the finite element approximation $u_{h}$, the computation of the bounds starts by solving the adjoint problem using finite elements. In general, both finite element approximations are computed using the same interpolation degree $p$, and thus, the first step of the bounding procedure has the same cost as the primal problem. Given the primal and adjoint finite element approximations, in the flux-free approach a local problem for each star is solved with a constant cost that only depends on the interpolation polynomial degree $q$ of the dual estimates and in the hybrid-flux approach first a local problem for each star is solved with a constant cost that only depends on the interpolation polynomial degree $p$ and then a local problem for each element is solved with a constant cost that only depends on the interpolation polynomial degree $q$ for the dual estimates.

Both approaches require looping on the vertex nodes of the mesh and the hybrid-flux approach requires and extra loop on the elements of the mesh. The cost of the vertex loop for the flux-free strategy is more expensive than the same loop for the hybrid-flux approach, since the unknowns for the flux-free local problems are directly the dual estimates (both in the edges and interior of the triangles) while the unknowns for the hybrid-flux approach in the first vertex-loop stage are the equilibrated fluxes (polynomials of degree $p$ at the edges of the elements incident to the node). During the second stage, the hybrid-flux approach unknowns are also the dual estimates of interpolation degree $q$ but the advantage is that the problems are solved independently on each element of the mesh.

Although the cost of the flux-free technique is slightly higher, numerical examples show that the use of flux-free techniques yields tighter bounds for the quantities of interest. Increasing the local interpolation degree $q$ in both approaches improves the bounds since the dual estimates have more degrees of freedom that can be used to optimize the bounds. However, based on the authors experience, there is no considerable gain in increasing the interpolation degree $q$, especially in the flux-free context, see [20]. Thus, it is advisable to use the least possible interpolation degree $q$ in both approaches, also in the hybrid-flux approach, since the quality of the bounds is mainly governed by the quality of the equilibrated fluxes and not by the interpolation degree $q$. Even if the local interpolation degree $q$ is increased in the hybrid-flux approach, in general this approach is not able to achieve the accuracy of the flux-free approach, thus, increasing the computational cost does not yield to the same accuracy. 


\section{BOUNDS FOR THE QUANTITY OF INTEREST $s=\ell^{\mathcal{O}}(u)$ : AN ALGORITHMIC SUMMARY}

According to theorem 1 upper and lower bounds of $s=\ell^{\mathcal{O}}(u)$ are available once upper bounds of the energy norm $\|z\|$ are obtained for the two combinations $(\alpha, \beta)=(\kappa, 1 / \kappa)$ and $(\alpha, \beta)=(\kappa,-1 / \kappa)$. The general strategy to obtain these upper bounds is devised in the previous section. Due to the linearity of the problem, obtaining the estimates for these two values $z=\kappa e^{\mathrm{s}} \pm 1 / \kappa \varepsilon^{\mathrm{s}}$ is equivalent to obtain the estimates for $z=e^{\mathrm{s}}$ and $z=\varepsilon^{\mathrm{s}}$ separately, that is for the two combinations $(\alpha, \beta)=(1,0)$ and $(\alpha, \beta)=(0,1)$.

This section summarizes the main steps to compute bounds for $\ell^{\mathcal{O}}(u)$ for both the flux-free and the hybrid-flux approach.

\subsection{Computation of the output bounds using the flux-free approach}

The main steps of the procedure to compute bounds for $\ell^{\mathcal{O}}(u)$ using the flux-free approach are the following:

1. Compute the primal and adjoint SUPG approximations, $u_{h}$ and $\psi_{h}$ respectively.

2. For each star $\omega^{i}$ (associated with node $\boldsymbol{x}^{i}$ of the mesh) compute the primal and adjoint dual estimates $\hat{\boldsymbol{q}}_{P}^{i}, \hat{\boldsymbol{q}}_{D}^{i} \in\left[\widehat{\mathbb{P}}^{q}\left(\omega^{i}\right)\right]^{2}$ and $\hat{r}_{P}^{i}, \hat{r}_{D}^{i} \in \widehat{\mathbb{P}}^{q}\left(\omega^{i}\right)$ such that for all $v \in \mathcal{V}\left(\omega^{i}\right)$

$$
\begin{aligned}
\int_{\omega^{i}}\left[\nu \hat{\boldsymbol{q}}_{P}^{i} \cdot \nabla v+\tilde{\sigma} \hat{r}_{P}^{i} v\right] \mathrm{d} \Omega & +\frac{1}{2} \int_{\Gamma_{\mathrm{N}} \cap \partial \omega^{i}} \boldsymbol{\alpha} \cdot \boldsymbol{n} \hat{r}_{P}^{i} v \mathrm{~d} \Gamma \\
& =R^{\mathrm{P}}\left(\phi^{i} v\right)-\sum_{\Omega_{k} \subset \omega^{i}} \int_{\Omega_{k}} \tau_{k}^{P} \mathcal{R}^{P}\left(u_{h}\right) \boldsymbol{\alpha} \cdot \boldsymbol{\nabla} \phi^{i} v d \Omega
\end{aligned}
$$

and

$$
\begin{aligned}
\int_{\omega^{i}}\left[\nu \hat{\boldsymbol{q}}_{D}^{i} \cdot \boldsymbol{\nabla} v+\tilde{\sigma} \hat{r}_{D}^{i} v\right] \mathrm{d} \Omega & +\frac{1}{2} \int_{\Gamma_{\mathrm{N}} \cap \partial \omega^{i}} \boldsymbol{\alpha} \cdot \boldsymbol{n} \hat{r}_{D}^{i} v \mathrm{~d} \Gamma \\
& =R^{\mathrm{D}}\left(\phi^{i} v\right)+\sum_{\Omega_{k} \subset \omega^{i}} \int_{\Omega_{k}} \tau_{k}^{D} \mathcal{R}^{D}\left(\psi_{h}\right) \boldsymbol{\alpha} \cdot \boldsymbol{\nabla} \phi^{i} v d \Omega .
\end{aligned}
$$

3 Recover the global estimates

$$
\hat{\boldsymbol{q}}_{P}=\sum_{i=1}^{n_{\mathrm{np}}} \hat{\boldsymbol{q}}_{P}^{i}, \quad \hat{r}_{P}=\sum_{i=1}^{n_{\mathrm{np}}} \hat{r}_{P}^{i} \quad \text { and } \quad \hat{\boldsymbol{q}}_{D}=\sum_{i=1}^{n_{\mathrm{np}}} \hat{\boldsymbol{q}}_{D}^{i}, \quad \hat{r}_{D}=\sum_{i=1}^{n_{\mathrm{np}}} \hat{r}_{D}^{i} .
$$

4 Compute the three scalar quantities

$$
\begin{aligned}
\left(\eta^{P}\right)^{2} & :=\sum_{k=1}^{n_{\mathrm{el}}} \eta_{k}^{P}=\sum_{k=1}^{n_{\mathrm{el}}} \int_{\Omega_{k}}\left[\nu \hat{\boldsymbol{q}}_{P} \cdot \hat{\boldsymbol{q}}_{P}+\tilde{\sigma}\left(\hat{r}_{P}\right)^{2}\right] \mathrm{d} \Omega+\frac{1}{2} \int_{\Gamma_{\mathrm{N}} \cap \Omega_{k}} \boldsymbol{\alpha} \cdot \boldsymbol{n}\left(\hat{r}_{P}\right)^{2} \mathrm{~d} \Gamma, \\
\left(\eta^{D}\right)^{2} & :=\sum_{k=1}^{n_{\mathrm{el}}} \eta_{k}^{D}=\sum_{k=1}^{n_{\mathrm{el}}} \int_{\Omega_{k}}\left[\nu \hat{\boldsymbol{q}}_{D} \cdot \hat{\boldsymbol{q}}_{D}+\tilde{\sigma}\left(\hat{r}_{D}\right)^{2}\right] \mathrm{d} \Omega+\frac{1}{2} \int_{\Gamma_{\mathrm{N}} \cap \Omega_{k}} \boldsymbol{\alpha} \cdot \boldsymbol{n}\left(\hat{r}_{D}\right)^{2} \mathrm{~d} \Gamma, \\
\eta^{P D} & :=\sum_{k=1}^{n_{\mathrm{el}}} \eta_{k}^{P D}=\sum_{k=1}^{n_{\mathrm{el}}} \int_{\Omega_{k}}\left[\nu \hat{\boldsymbol{q}}_{P} \cdot \hat{\boldsymbol{q}}_{D}+\tilde{\sigma} \hat{r}_{P} \hat{r}_{D}\right] \mathrm{d} \Omega+\frac{1}{2} \int_{\Gamma_{\mathrm{N}} \cap \Omega_{k}} \boldsymbol{\alpha} \cdot \boldsymbol{n} \hat{r}_{P} \hat{r}_{D} \mathrm{~d} \Gamma,
\end{aligned}
$$

5. Recover the bounds for the output $s^{l b} \leq s \leq s^{u b}$ as

$$
s^{l b}:=s_{h}+R^{\mathrm{P}}\left(\psi_{h}\right)-\frac{1}{2} \eta^{P} \eta^{D}+\frac{1}{2} \eta^{P D} \leq s \leq s_{h}+R^{\mathrm{P}}\left(\psi_{h}\right)+\frac{1}{2} \eta^{P} \eta^{D}+\frac{1}{2} \eta^{P D}=: s^{u b},
$$

where $s_{h}=\ell^{\mathcal{O}}\left(u_{h}\right)$. 


\subsection{Computation of the output bounds using the hybrid-flux approach}

The main steps of the procedure to compute bounds for $\ell^{\mathcal{O}}(u)$ using the hybrid-flux approach are the following:

1. Compute the primal and adjoint SUPG approximations, $u_{h}$ and $\psi_{h}$ respectively.

2. Compute $\lambda^{P}$ and $\lambda^{D}$ solutions of

$$
R^{\mathrm{P}}(v)-\sum_{k=1}^{n_{\mathrm{el}}} \int_{\Omega_{k}} \tau_{k}^{P} \mathcal{R}^{P}\left(u_{h}\right) \boldsymbol{\alpha} \cdot \nabla v d \Omega+\sum_{\gamma \in \Gamma_{h}} \int_{\gamma} \lambda^{P}[v] \mathrm{d} \Gamma=0 \quad \forall v \in \widehat{\mathcal{V}}^{h},
$$

and

$$
R^{\mathrm{D}}(v)+\sum_{k=1}^{n_{\mathrm{el}}} \int_{\Omega_{k}} \tau_{k}^{D} \mathcal{R}^{D}\left(\psi_{h}\right) \boldsymbol{\alpha} \cdot \boldsymbol{\nabla} v d \Omega+\sum_{\gamma \in \Gamma_{h}} \int_{\gamma} \lambda^{D}[v] \mathrm{d} \Gamma=0 \quad \forall v \in \widehat{\mathcal{V}}^{h} .
$$

3. For each element of the mesh $\Omega_{k}$ compute the primal and adjoint dual estimates $\hat{\boldsymbol{q}}_{P}^{k}, \hat{\boldsymbol{q}}_{D}^{k} \in$ $\left[\mathbb{P}^{q}\left(\Omega_{k}\right)\right]^{2}$ and $\hat{r}_{P}^{k}, \hat{r}_{D}^{k} \in \mathbb{P}^{q}\left(\Omega_{k}\right)$ such that for all $v \in \mathcal{H}^{1}\left(\Omega_{k}\right)$

$$
\int_{\Omega_{k}}\left[\nu \hat{\boldsymbol{q}}_{P}^{k} \cdot \boldsymbol{\nabla} v+\tilde{\sigma} \hat{r}_{P}^{k} v\right] \mathrm{d} \Omega+\frac{1}{2} \int_{\Gamma_{\mathrm{N}} \cap \partial \Omega_{k}} \boldsymbol{\alpha} \cdot \boldsymbol{n} \hat{r}_{P}^{k} v \mathrm{~d} \Gamma=R_{k}^{P}(v)+\int_{\partial \Omega_{k} \backslash \Gamma_{\mathrm{N}}} \varsigma_{k} \lambda^{P} v \mathrm{~d} \Gamma,
$$

and

$$
\int_{\Omega_{k}}\left[\nu \hat{\boldsymbol{q}}_{D}^{k} \cdot \nabla v+\tilde{\sigma} \hat{r}_{D}^{k} v\right] \mathrm{d} \Omega+\frac{1}{2} \int_{\Gamma_{\mathrm{N}} \cap \partial \Omega_{k}} \boldsymbol{\alpha} \cdot \boldsymbol{n} \hat{r}_{D}^{k} v \mathrm{~d} \Gamma=R_{k}^{D}(v)+\int_{\partial \Omega_{k} \backslash \Gamma_{\mathrm{N}}} \varsigma_{k} \lambda^{D} v \mathrm{~d} \Gamma .
$$

3 Compute the three scalar quantities

$$
\begin{aligned}
&\left(\eta^{P}\right)^{2}:=\sum_{k=1}^{n_{\mathrm{el}}} \eta_{k}^{P}=\sum_{k=1}^{n_{\mathrm{el}}} \int_{\Omega_{k}}\left[\nu \hat{\boldsymbol{q}}_{P} \cdot \hat{\boldsymbol{q}}_{P}+\tilde{\sigma}\left(\hat{r}_{P}\right)^{2}\right] \mathrm{d} \Omega+\frac{1}{2} \int_{\cap \Omega_{k}} \boldsymbol{\alpha} \cdot \boldsymbol{n}\left(\hat{r}_{P}\right)^{2} \mathrm{~d} \Gamma, \\
&\left(\eta^{D}\right)^{2}:=\sum_{k=1}^{n_{\mathrm{el}}} \eta_{k}^{D}=\sum_{k=1}^{n_{\mathrm{el}}} \int_{\Omega_{k}}\left[\nu \hat{\boldsymbol{q}}_{D} \cdot \hat{\boldsymbol{q}}_{D}+\tilde{\sigma}\left(\hat{r}_{D}\right)^{2}\right] \mathrm{d} \Omega+\frac{1}{2} \int_{\cap \Omega_{k}} \boldsymbol{\alpha} \cdot \boldsymbol{n}\left(\hat{r}_{D}\right)^{2} \mathrm{~d} \Gamma, \\
& \eta^{P D}:=\sum_{k=1}^{n_{\mathrm{el}}} \eta_{k}^{P D}=\sum_{k=1}^{n_{\mathrm{el}}} \int_{\Omega_{k}}\left[\nu \hat{\boldsymbol{q}}_{P} \cdot \hat{\boldsymbol{q}}_{D}+\tilde{\sigma} \hat{r}_{P} \hat{r}_{D}\right] \mathrm{d} \Omega+\frac{1}{2} \int_{\cap \Omega_{k}} \boldsymbol{\alpha} \cdot \boldsymbol{n} \hat{r}_{P} \hat{r}_{D} \mathrm{~d} \Gamma,
\end{aligned}
$$

4. Recover the bounds for the output $s^{l b} \leq s \leq s^{u b}$ as

$$
s^{l b}:=s_{h}+R^{\mathrm{P}}\left(\psi_{h}\right)-\frac{1}{2} \eta^{P} \eta^{D}+\frac{1}{2} \eta^{P D} \leq s \leq s_{h}+R^{\mathrm{P}}\left(\psi_{h}\right)+\frac{1}{2} \eta^{P} \eta^{D}+\frac{1}{2} \eta^{P D}=: s^{u b},
$$

where $s_{h}=\ell^{\mathcal{O}}\left(u_{h}\right)$.

\section{NUMERICAL EXAMPLES}

This section presents the performance of the estimates providing the bounds for quantities of interest in three numerical examples, which are defined in a two-dimensional domain and which are discretized using conforming piecewise linear finite elements.

In all the examples, both the primal and adjoint approximations $u_{h}$ and $\psi_{h}$ are computed both using the standard Galerkin Finite Element method and the SUPG method. When using stabilization techniques, as the SUPG method, the choice of the stabilization parameter plays a major role, since the accuracy of the discrete solution is highly influenced by this choice. The appropiate selection of 
this parameter is not discussed here since the primary goal of this work is to show the performance of the error estimation strategy. Thus, the stabilization parameter is chosen following [22]. However, the error estimation procedure is valid for any choice of the definition of the stabilization parameter, see for instance [23, 24, 25, 26].

The stabilization parameter for the primal approximation $u_{h}$ is taken to be constant inside each element $\Omega_{k}$ of the mesh,

$$
\tau_{k}^{P}=\frac{h_{k}}{2|\boldsymbol{\alpha}|_{k}}\left(1+\frac{9}{(P e)_{k}^{2}}+\left(\frac{h_{k} \sigma_{k}}{2|\boldsymbol{\alpha}|_{k}}\right)^{2}\right)^{-\frac{1}{2}}
$$

where $h_{k}$ is the element size - computed as the radius of the circumcircle of the triangle -, $|\boldsymbol{\alpha}|_{k}$ is a measure of the norm of the velocity $\alpha$ inside the element - computed as the norm of the velocity at the barycenter of the triangle -, and $P e_{k}$ is the local Péclet number defined as:

$$
(P e)_{k}=\frac{1}{2}|\boldsymbol{\alpha}|_{k} h_{k} \nu_{k}
$$

Analogously, the stabilization parameter for the adjoint problem is

$$
\tau_{k}^{D}=\frac{h_{k}}{2|\boldsymbol{\alpha}|_{k}}\left(1+\frac{9}{(P e)_{k}^{2}}+\left(\frac{h_{k}\left(\sigma_{k}-|\nabla \cdot \boldsymbol{\alpha}|_{k}\right)}{2|\boldsymbol{\alpha}|_{k}}\right)^{2}\right)^{-\frac{1}{2}} .
$$

Note that if the velocity field $\alpha$ is divergence free, then the primal and adjoint stabilization parameters coincide, $\tau_{k}^{P}=\tau_{k}^{D}$.

As mentioned above, in the following examples both the Galerkin and SUPG approximations of the problem are computed using linear elements, that is, the parameter describing the space discretization is $p=1$, and the dual estimates providing the bounds for the output are computed using piecewise third order polynomials, which corresponds to $q=3$. The dual estimates are computed both using the flux-free and the hybrid-flux error estimation strategies (the later also called residual equilibrated method). In the following, the notation FF and EQ is used in figures and tables to denote the two previous techniques respectively.

In the following, the bound average $s^{a v e}:=\left(s^{u b}+s^{l b}\right) / 2$ is taken as a new approximation of the quantity of interest and the half bound gap $\Delta=\left(s^{u b}-s^{l b}\right) / 2$ is seen as an error indicator. Note that stating that $s^{l b}$ and $s^{u b}$ are exact upper bounds for the output $s$ implies that $s \in\left(s^{l b}, s^{u b}\right)$ which can be rewritten as $s=s^{\text {ave }} \pm \Delta$.

The meshes are adapted to reduce the half bound gap $\Delta$. In the examples a simple adaptive strategy is used based on the decomposition of $\Delta$ into local positive contributions from the elements:

$$
\Delta=\sum_{k=1}^{n_{\mathrm{el}}} \Delta_{k}
$$

where the element contribution to the half bound gap $\Delta_{k}$ is

$$
\Delta_{k}:=\frac{1}{4} \kappa^{2} \eta_{k}^{P}+\frac{1}{4 \kappa^{2}} \eta_{k}^{D}
$$

Note that this decomposition is valid because

$$
\Delta=\frac{s^{u b}-s^{l b}}{2}=\frac{1}{2} \eta^{P} \eta^{D}=\frac{1}{4} \kappa^{2}\left(\eta^{P}\right)^{2}+\frac{1}{4 \kappa^{2}}\left(\eta^{D}\right)^{2}=\sum_{k=1}^{n_{\mathrm{el}}}\left[\frac{1}{4} \kappa^{2} \eta_{k}^{P}+\frac{1}{4 \kappa^{2}} \eta_{k}^{D}\right]=\sum_{k=1}^{n_{\mathrm{el}}} \Delta_{k} .
$$

The remeshing strategy consists in subdividing the elements with the larger values of $\Delta_{k}$ at each step of the adaptive procedure. 


\subsection{Example 1: quasi-2D transport}

The first example is the quasi-2D transport problem introduced in [10]. The advection-diffusion equation is considered in the unit square $\Omega=[0,1] \times[0,1]$ with $\nu=1, \sigma=0$ and a uniform horizontal velocity field $\boldsymbol{\alpha}=(\alpha, 0)$. The boundary conditions are of Dirichlet type on the lateral sides, $u(1, y)=0$ and $u(0, y)=1$, and Neumann homogeneous on the top and bottom sides. The source term is $f=0$ so that the analytical solution is

$$
u(x, y)=\frac{e^{\alpha}-e^{\alpha x}}{e^{\alpha}-1}
$$

and the quantity of interest is taken to be the average normal gradient on the right side of the domain, namely

$$
s=\int_{0}^{1} \nabla u(1, y) \cdot \boldsymbol{n} \mathrm{d} \Gamma=\frac{\alpha e^{\alpha}}{1-e^{\alpha}} .
$$

Following [10], this quantity of interest can be rewritten using the interior function $\chi=x$ as $s=a(u, \chi)$, which in turn using the Green's formula can be rewritten as $s=\ell^{\mathcal{O}}(u)$ using the functional

$$
\ell^{\mathcal{O}}(v)=a(v, \chi) .
$$

This quantity of interest is not directly in the form of (5), but using Green's formula, $a(v, \chi)$ can be rewritten like (5) with $f^{\mathcal{O}}=-\nabla \cdot(\nu \nabla \chi)-\nabla \cdot \boldsymbol{\alpha} \chi-\boldsymbol{\alpha} \cdot \nabla \chi+\sigma \chi$ and $g^{\mathcal{O}}=\nu \boldsymbol{\nabla} \chi \cdot \boldsymbol{n}+\boldsymbol{\alpha} \cdot \boldsymbol{n} \chi$ for all $v \in \mathcal{V}$. However, it is worth noting that following the derivations included in [3] it is possible to compute the dual estimates without doing the conversion of the functional $\ell^{\mathcal{O}}(v)$ in terms of $f^{\mathcal{O}}$ and $g^{\mathcal{O}}$, in a much simpler manner.

This example allows testing the quality of the bounds for the output for different values of $\alpha$, ranging from a pure diffusion problem to a advection-dominated advection-diffusion problem. Four different strategies are compared for the values of $\alpha=5,150$ and 500: the bounds obtained for the stabilized hybrid-flux and flux-free strategies presented in this paper are compared with the bounds obtained using the standard hybrid-flux and flux-free strategies presented in [10] and [1] respectively. The results are shown in figure 1 and table I.

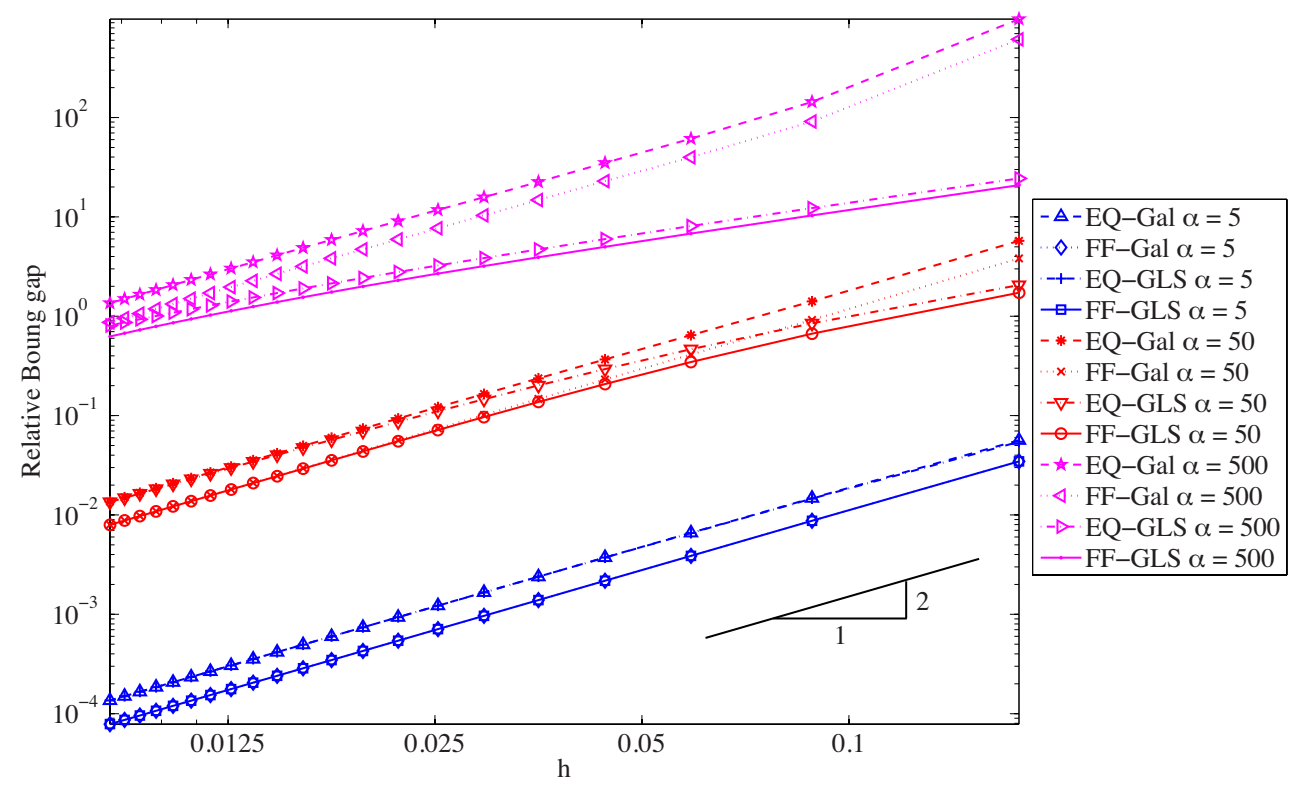

Figure 1. Example 1: convergence of the relative half bound gap $(\Delta / s)$ for a uniform $h$-refinement procedure obtained from standard Galerkin finite element approximations and SUPG approximations using both hybrid-flux and flux-free strategies. 


\begin{tabular}{|c|c|c|c|c|c|c|c|}
\hline & \multicolumn{2}{|c|}{$\begin{array}{c}\alpha=5 \\
s=-5.033918\end{array}$} & \multicolumn{2}{|c|}{$\begin{array}{c}\alpha=50 \\
s=-50\end{array}$} & \multicolumn{2}{|c|}{$\begin{array}{c}\alpha=500 \\
s=-500\end{array}$} \\
\hline \multirow{6}{*}{ 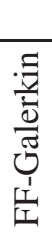 } & $n_{\mathrm{el}}$ & $s^{\text {ave }}$ & $\Delta /\left|s^{\text {ave }}\right|$ & $s^{a v e}$ & $\Delta /\left|s^{\text {ave }}\right|$ & $s^{\text {ave }}$ & $\Delta /\left|s^{\text {ave }}\right|$ \\
\hline & 32 & -5.02103 & 0.03474 & 13.85636 & 13.80392 & 273561.10163 & 1.11887 \\
\hline & 1152 & -5.03362 & 0.00097 & -50.00000 & 0.10165 & -330.48485 & 15.68297 \\
\hline & 3872 & -5.03383 & 0.00029 & -50.00000 & 0.02963 & -499.99731 & 3.17566 \\
\hline & 8192 & -5.03388 & 0.00014 & -50.00000 & 0.01384 & -500.00000 & 1.50201 \\
\hline & 14112 & -5.03389 & 0.00008 & -50.00000 & 0.00798 & -500.00000 & 0.86799 \\
\hline \multirow{5}{*}{ 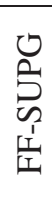 } & 32 & -5.01987 & 0.03470 & -50.08000 & 1.72392 & -507.69541 & 20.57684 \\
\hline & 1152 & -5.03362 & 0.00097 & -50.00000 & 0.09685 & -500.00000 & 3.20511 \\
\hline & 3872 & -5.03383 & 0.00029 & -50.00000 & 0.02926 & -500.00000 & 1.55460 \\
\hline & 8192 & -5.03388 & 0.00014 & -50.00000 & 0.01377 & -500.00000 & 0.93795 \\
\hline & 14112 & -5.03389 & 0.00008 & -50.00000 & 0.00796 & -500.00000 & 0.62550 \\
\hline \multirow{5}{*}{ 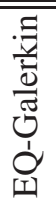 } & 32 & -5.02872 & 0.05664 & 42.04600 & 6.81851 & 4337796.96760 & $\bar{~} 1.11699$ \\
\hline & 1152 & -5.03379 & 0.00166 & -49.99259 & 0.16532 & -245.33712 & 32.20248 \\
\hline & 3872 & -5.03388 & 0.00050 & -49.99772 & 0.04958 & -499.78202 & 4.88044 \\
\hline & 8192 & -5.03390 & 0.00023 & -49.99891 & 0.02350 & -499.89784 & 2.32843 \\
\hline & 14112 & -5.03391 & 0.00014 & -49.99936 & 0.01366 & -499.94023 & 1.35665 \\
\hline \multirow{5}{*}{ 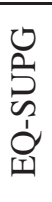 } & 32 & -5.03205 & 0.05456 & -50.27436 & 2.04817 & -546.27178 & 22.33189 \\
\hline & 1152 & -5.03379 & 0.00166 & -49.99375 & 0.14623 & -499.66067 & 3.85809 \\
\hline & 3872 & -5.03388 & 0.00050 & -49.99788 & 0.04753 & -499.88878 & 1.90775 \\
\hline & 8192 & -5.03390 & 0.00023 & -49.99895 & 0.02302 & -499.94213 & 1.18005 \\
\hline & 14112 & -5.03391 & 0.00014 & -49.99937 & 0.01349 & -499.96335 & 0.80898 \\
\hline
\end{tabular}

Table I. Example 1: bounds for a uniform $h$-refinement procedure obtained from standard Galerkin finite element approximations and SUPG approximations for different values of $\alpha=5,50,500$.

Figure 1 shows the convergence of the half bound gap. As expected, the half bound gap has a quadratic rate of converge in all the strategies, although this convergence rate is only achieved in the asymptotic range. It can be appreciated that as the influence of the convective term becomes more important, finer meshes are needed to reach the asymptotic range.

As noted in [1], the results herein confirm that the flux-free strategy has a better performance than the hybrid-flux strategy, both for standard and stabilized formulations. Also, it can be seen that for low values of the advection parameter the bounds obtained using the standard Galerkin method are pretty similar to the ones obtained using stabilized methods. However, as the advection parameter increases, the stabilized formulations perform better than the non-stabilized ones. As observed in [10] and [1] as the advection parameter increases the bounds degenerate due to the introduction of the symmetrized residual equations. As it can be seen, the use of stabilization techniques does not avoid the blow-up of the bounds for highly dominated advection problems, but it allows alleviating this behavior for intermediate values of $\alpha$. Finally, it is worth noting that as the finite element mesh is refined, the difference between the performance of standard and stabilized formulations diminishes and both approaches provide similar results, as expected.

The performance of the bounds in an adaptive process is analyzed for the value $\alpha=500$. Starting with a structured mesh of 64 triangular elements, a series of adapted meshes is produced by subdividing at each step the elements whose contribution to the half bound gap is larger than the average contribution, that is, $\Delta_{k}>\Delta / n_{\mathrm{el}}$. The adaptive procedure is guided by the indicators (local half bound gap) provided by the strict flux-free error estimate, but at each step, the bounds provided by the strict hybrid-flux strategy are also computed to compare the results. The initial mesh of 64 elements certifies a wide interval for the quantity of interest $s=20165.45 \pm 131.51 \%$ using the standard Galerkin approach and $s=-499.99 \pm 1271.33 \%$ using the SUPG approach. After remeshing, the bounds associated with the final mesh set a much narrower interval $s=-500.00 \pm$ 
$1.39 \%$ (for the standard Galerkin approach for a mesh of 11422 elements) and $s=-500.00 \pm 0.72 \%$ (for the SUPG approach for a mesh of 13280 elements). The results for the intermediate meshes can be seen in figure 2. It can be observed that stabilizing the solutions for large Péclet numbers helps

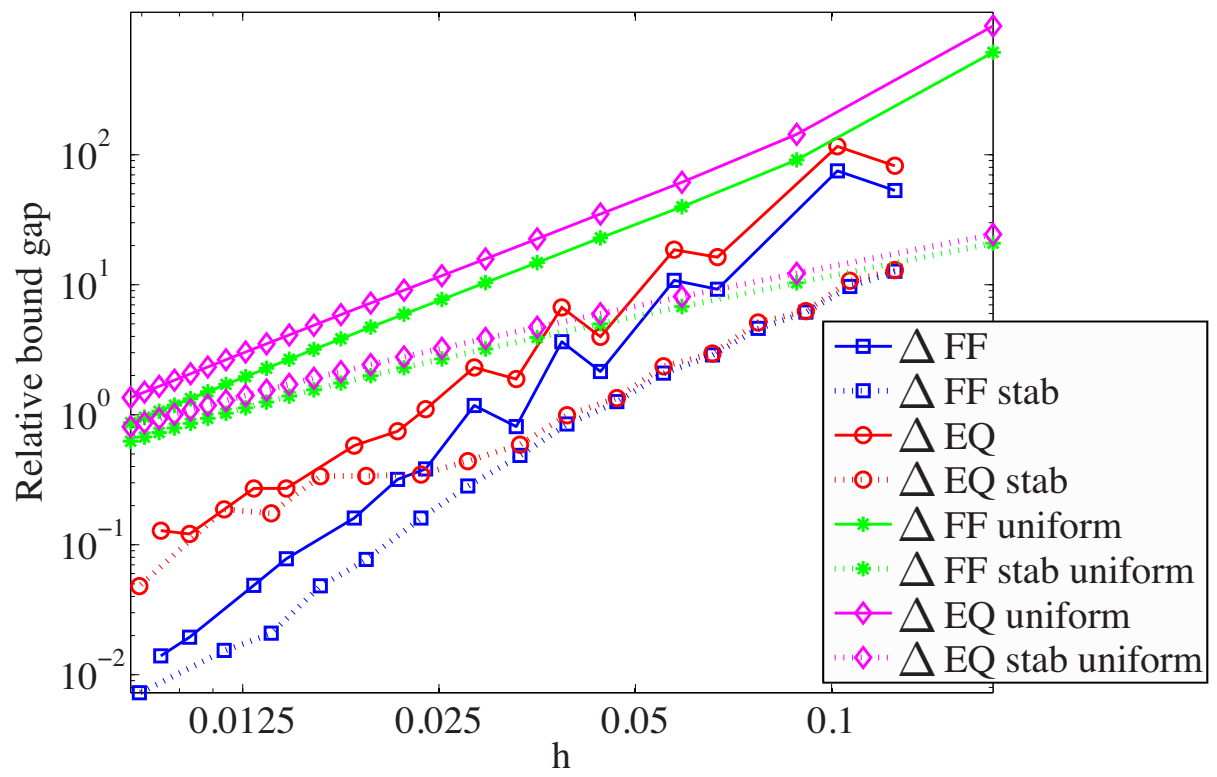

Figure 2. Example 1: convergence of the relative half bound gap $(\Delta /|s|)$ for an adaptive $h$-refinement procedure obtained from standard Galerkin finite element approximations and SUPG approximations.

Comparison with the results for the uniform mesh refinement.

reducing the bound gap with no additional cost both for the hybrid-flux and flux-free approaches. Figure 3 shows the final adapted meshes obtained for both the Galerkin and SUPG approaches. The meshes are refined in the areas where either the primal or adjoint solutions present the boundary layers. The main difference between both approaches is that in the first iterations the Galerkin method yields a highly oscillating solution which produces the refinement in areas where no refinement is needed (interior of the square).
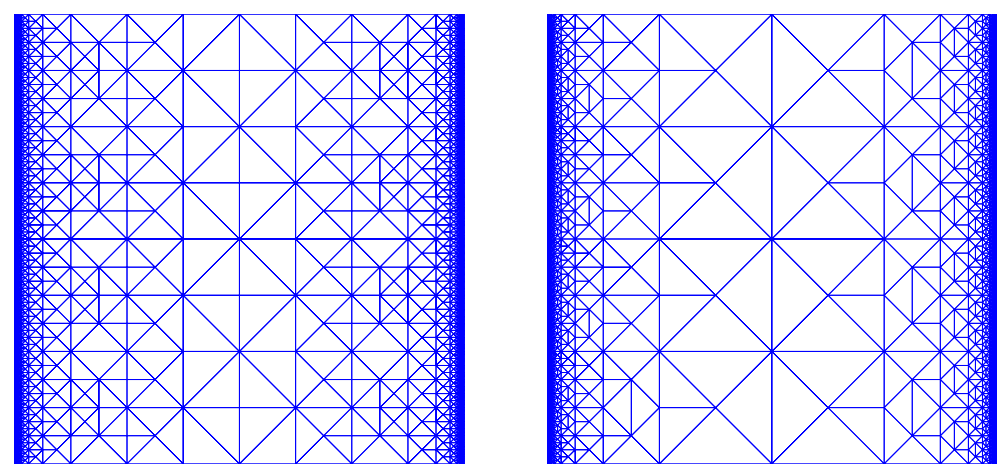

Figure 3. Example 1: Final adapted meshes obtained for both the Galerkin (left) and SUPG (right) approaches consisting of 11422 and 13280 elements respectively.

\subsection{Example 2: interior layers behind an obstacle}

The second example is taken from [27]. The computational domain is

$$
\Omega=\left\{(x, y) \in(-1,1)^{2},|x|+|y|>1 / 2\right\} .
$$


where the hole inside the square is conceived as an obstacle inside the computational domain (see figure 4). Equation (2) is solved in $\Omega$ with $\nu=1, \sigma=0$ and a uniform horizontal velocity field $\boldsymbol{\alpha}=(300,0)$. The boundary conditions are of Dirichlet type on all the boundary, homogeneous in the outer square and equal to 1 in the interior square, that is

$$
u_{D}= \begin{cases}1 & \text { for }|x|+|y|=1 / 2 \\ 0 & \text { elsewhere }\end{cases}
$$
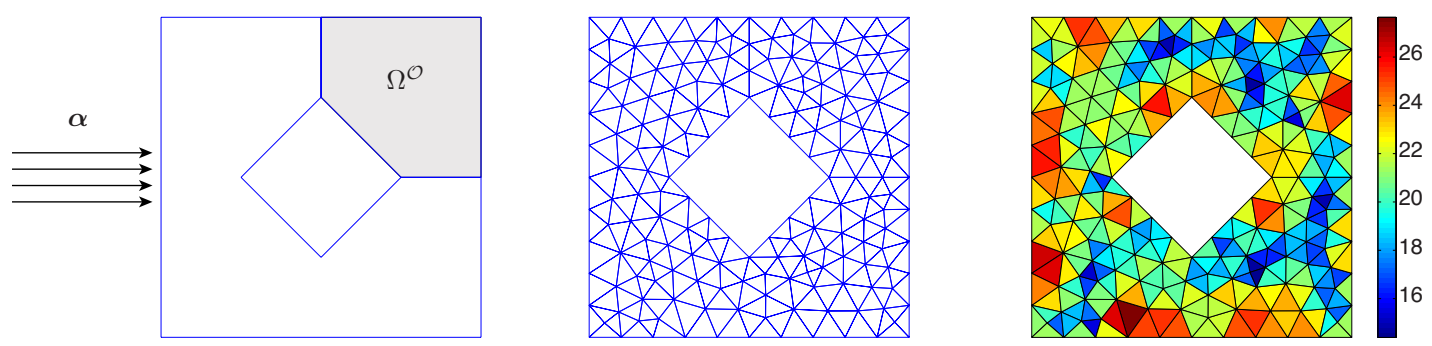

Figure 4. Example 2: Domain (left), initial mesh (both for the uniform and adaptive refinements) consisting of 300 triangular linear elements (center) and local Péclet number distribution for the initial mesh (right).

The obstacle inside the flow field gives rise to two interior layers and a boundary layer at the front part of the obstacle (with respect to the flow) and a boundary layer at a part of the boundary behind the obstacle.

The quantity of interest is the integral of the solution in the region $\Omega^{\mathcal{O}} \in \Omega \cap[0,1]^{2}$ which corresponds to $f^{\mathcal{O}}=1$ in $\Omega^{\mathcal{O}}$ and zero elsewhere.

The quality of the bounds is analyzed first for a uniform mesh refinement. The primal and adjoint solutions obtained with the mesh of 8012 elements are shown in figure 5. As it can be seen, neither the Galerkin nor the SUPG manage to properly supress the spurious local oscillations appearing in the discrete solutions for this quite fine uniformly-refined mesh. However, even though the proposed stabilization technique does not completely remove the spurious oscillations, the SUPG method provides a much more accurate solution than the Galerkin method.

The results of the a-posteriori error estimates presented in this paper are displayed in table II and in figure 6. As it can be seen, for coarse meshes the use of stabilization techniques provides

\begin{tabular}{|c|c|c|c|c|c|c|c|c|}
\hline & \multicolumn{3}{|c|}{ flux-free } & \multicolumn{3}{|c|}{ hybrid-flux } \\
\hline & $n_{\mathrm{el}}$ & $s_{h}$ & $s^{l b}$ & $s^{u b}$ & $\Delta$ & $s^{l b}$ & $s^{u b}$ & $\Delta$ \\
\hline \multirow{5}{*}{$\frac{.}{\frac{\vec{d}}{\mathbb{d}}}$} & 300 & .429511 & -20.630848 & 17.672803 & 19.151825 & -37.282079 & 31.544310 & 34.413194 \\
\hline & 74 & & -7.343591 & 8.283259 & 813 & -15.776 & 16.418931 & 16.097538 \\
\hline & 1694 & & -3.685 & 4.320300 & 4.003 & -8.935 & 9.011232 & 8.973247 \\
\hline & 3725 & & -1.8696 & 2.650419 & 2. & -5.05 & 5.745099 & 5.401981 \\
\hline & 8012 & 0.39 & -0.94 & 1.691 & & $-3.0^{\prime}$ & 3.685708 & 3.380092 \\
\hline \multirow{5}{*}{ 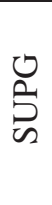 } & 300 & 0.407135 & -5.336663 & 6.048435 & 5.692549 & -11.895049 & 11.616651 & 11.755850 \\
\hline & 744 & 0.427548 & -3.941503 & 4.680051 & 4.310777 & -9.416113 & 9.122311 & 9.269212 \\
\hline & 1694 & 0.419324 & -2.582214 & 3.321779 & 2.9519 & -6.698040 & 6.685539 & 6.691789 \\
\hline & 3725 & 0.40 & -1.584882 & 2.349696 & $1.967^{\circ}$ & -3.878484 & 4.349016 & 4.113750 \\
\hline & 8012 & 0.398523 & -0.878744 & 1.646389 & 1.262566 & -2.626193 & 3.182316 & 2.904255 \\
\hline
\end{tabular}

Table II. Example 2: bounds for a uniform $h$-refinement procedure obtained from standard Galerkin finite element approximations and SUPG approximations.

a clear reduction of the half bound gap that becomes less important as the finite element mesh is 

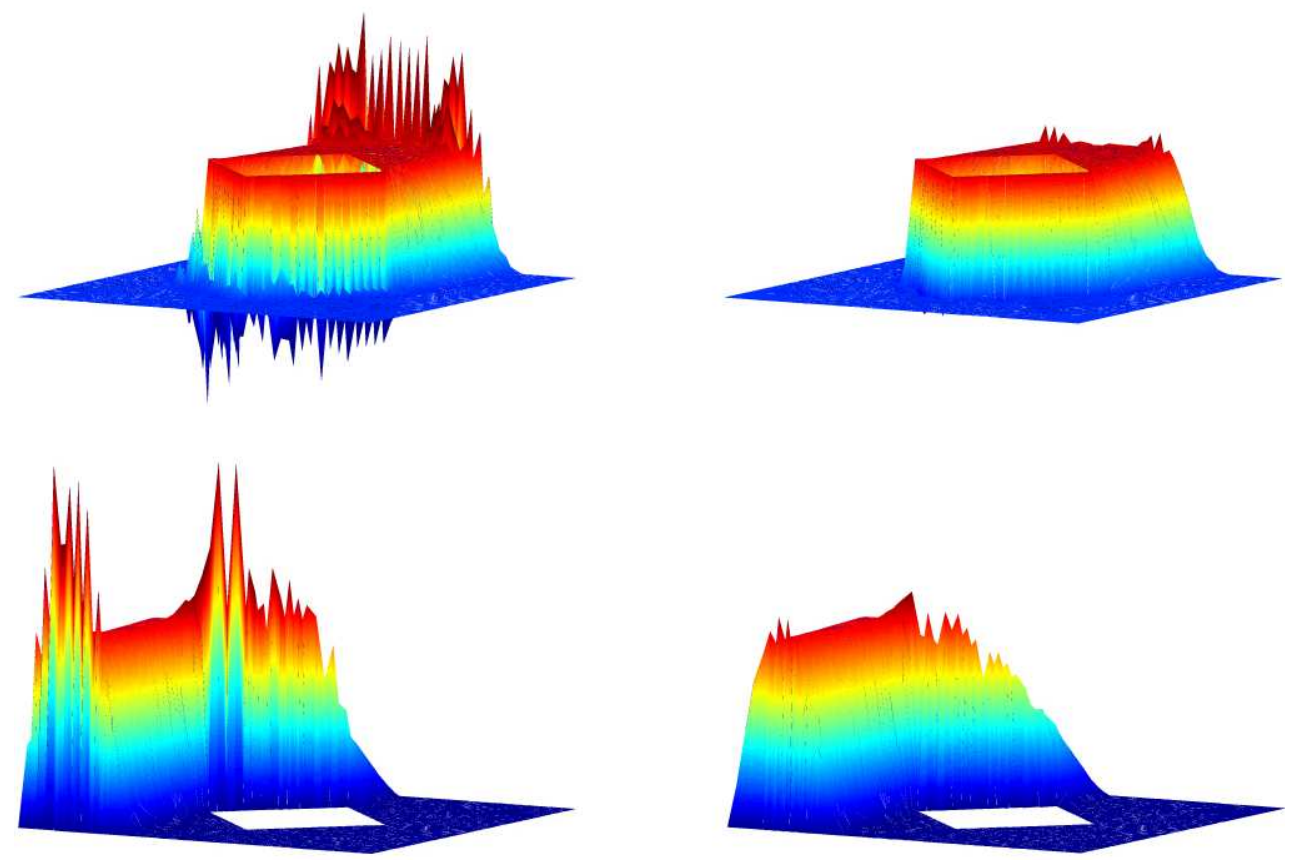

Figure 5. Example 2: Primal (top) and adjoint (bottom) solutions for the last mesh of the uniform refinement (consisting of 8012 elements) obtained using the standard Galerkin finite element method (left) and the SUPG method (right).
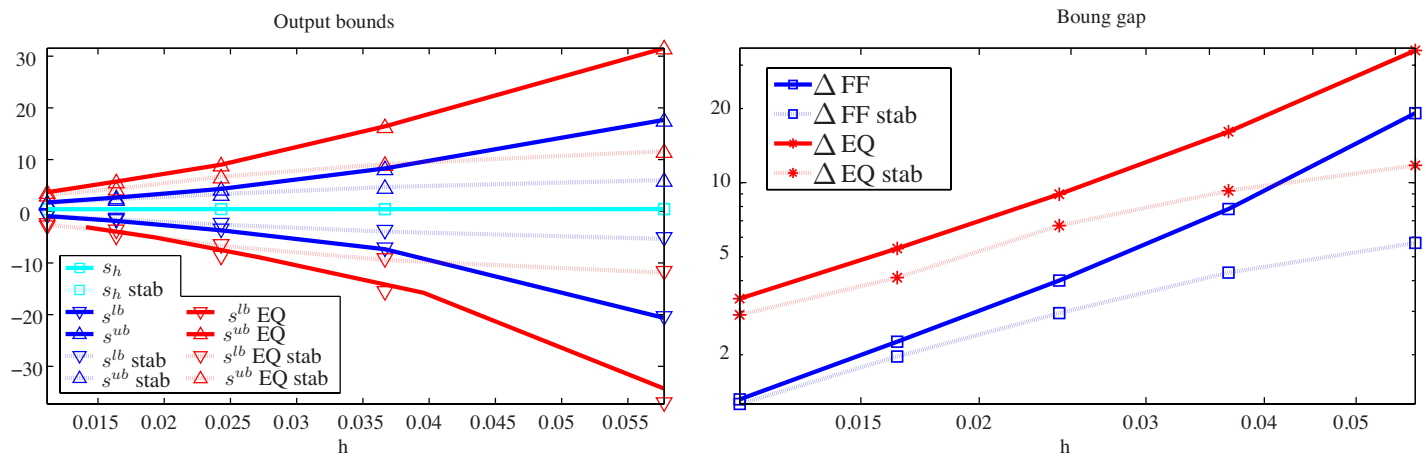

Figure 6. Example 2: bounds for a uniform $h$-refinement procedure obtained from standard Galerkin finite element approximations and SUPG approximations (left) and its convergence (right).

refined. Also, again the flux-free strategy provides better results than the hybrid-flux one. It can also be appreciated that the standard method to obtain bounds for quantities of interest, even when using stabilization strategies, yields poor results when using a uniform refinement (very fine meshes are needed to effectively reduce the bound gaps). Thus in this case it is crucial to use adaptive techniques.

The quality of the bounds is also analyzed for an adaptive refinement. A series of adapted meshes is produced by subdividing at each step $10 \%$ of the elements, those with the larger contributions to the half bound gap, until $\Delta<0.016$. The adaptive procedure is guided by the indicators (local half bound gap) provided by the strict flux-free error estimate. However, in each step, the bounds provided by the strict hybrid-flux strategy are also computed to compare the results. 
The initial mesh of 300 elements certifies a wide interval for the quantity of interest $s=$ $-1.479 \pm 19.152$ using the standard Galerkin approach and $s=0.356 \pm 5.693$ using the SUPG approach. After remeshing, the bounds associated with the final mesh set a much narrower interval $s=0.3798 \pm 0.01508$ (for the standard Galerkin approach for a mesh of 12126 elements) and $s=0.3800 \pm 0.01574$ (for the SUPG approach for a mesh of 12330 elements). The results for the intermediate meshes can be seen in tables III and IV and in figure 7.

standard Galerkin finite element approximation

\begin{tabular}{|c|c|c|c|c|c|c|c|}
\hline & & \multicolumn{3}{|c|}{ flux-free } & \multicolumn{3}{|c|}{ hybrid-flux } \\
\hline$n_{\mathrm{el}}$ & $s_{h}$ & $s^{l b}$ & $s^{u b}$ & $\Delta$ & $s^{l b}$ & $s^{u b}$ & $\Delta$ \\
\hline 300 & 0.429511 & -20.630848 & 17.672803 & 19.151825 & -37.282079 & 31.544310 & 34.413194 \\
\hline 357 & 0.431046 & -11.812368 & 10.758631 & 11.285499 & -24.099566 & 21.238144 & 22.668855 \\
\hline 415 & 0.394818 & -6.887437 & 9.467279 & 8.177358 & -15.872191 & 19.296528 & 17.584360 \\
\hline 492 & 0.401901 & -4.615369 & 5.155705 & 4.885537 & -11.592608 & 11.844514 & 11.718561 \\
\hline 577 & 0.399312 & -2.731070 & 3.449440 & 3.090255 & -8.311474 & 8.934480 & 8.622977 \\
\hline 682 & 0.399517 & -2.105179 & 2.619031 & 2.362105 & -7.025657 & 7.210070 & 7.117863 \\
\hline 794 & 0.392198 & -1.205988 & 1.968473 & 1.587230 & -5.302291 & 5.927614 & 5.614953 \\
\hline 923 & 0.381479 & -0.972389 & 1.666009 & 1.319199 & -4.565030 & 5.216576 & 4.890803 \\
\hline 1072 & 0.385116 & -0.704109 & 1.404819 & 1.054464 & -3.927010 & 4.565738 & 4.246374 \\
\hline 1230 & 0.384589 & -0.446016 & 1.198208 & 0.822112 & -3.183883 & 3.910679 & 3.547281 \\
\hline 1405 & 0.383902 & -0.224545 & 1.002875 & 0.613710 & -2.686555 & 3.452816 & 3.069686 \\
\hline 1615 & 0.382516 & -0.103822 & 0.868474 & 0.486148 & -2.229312 & 2.983397 & 2.606355 \\
\hline 1851 & 0.381386 & -0.007228 & 0.769074 & 0.388151 & -1.882978 & 2.636305 & 2.259642 \\
\hline 2115 & 0.381416 & 0.079254 & 0.683584 & 0.302165 & -1.677128 & 2.435176 & 2.056152 \\
\hline 2397 & 0.381370 & 0.138560 & 0.624934 & 0.243187 & -1.475130 & 2.227198 & 1.851164 \\
\hline 2768 & 0.380450 & 0.188917 & 0.572342 & 0.191713 & -1.380715 & 2.140712 & 1.760714 \\
\hline 3198 & 0.380351 & 0.239798 & 0.519340 & 0.139771 & -1.075553 & 1.832568 & 1.454060 \\
\hline 3575 & 0.380190 & 0.277870 & 0.481789 & 0.101959 & -0.789506 & 1.546283 & 1.167894 \\
\hline 4022 & 0.380264 & 0.293013 & 0.466865 & 0.086926 & -0.791676 & 1.550964 & 1.171320 \\
\hline 4580 & 0.379990 & 0.308917 & 0.450748 & 0.070915 & -0.704982 & 1.463868 & 1.084425 \\
\hline 5186 & 0.379945 & 0.320062 & 0.439756 & 0.059847 & -0.633929 & 1.391602 & 1.012765 \\
\hline 6116 & 0.379935 & 0.336028 & 0.423666 & 0.043819 & -0.415468 & 1.172784 & 0.794126 \\
\hline 6840 & 0.379865 & 0.347440 & 0.412151 & 0.032355 & -0.269414 & 1.027825 & 0.648620 \\
\hline 7895 & 0.379881 & 0.353315 & 0.406319 & 0.026502 & -0.230727 & 0.989450 & 0.610089 \\
\hline 8967 & 0.379837 & 0.357404 & 0.402180 & 0.022388 & -0.186034 & 0.944352 & 0.565193 \\
\hline 10301 & 0.379816 & 0.360808 & 0.398750 & 0.018971 & -0.156364 & 0.913923 & 0.535144 \\
\hline 12126 & 0.379826 & 0.364697 & 0.394854 & 0.015079 & -0.058975 & 0.817103 & 0.438039 \\
\hline
\end{tabular}

Table III. Example 2: bounds for an apative $h$-refinement procedure obtained from standard Galerkin finite element approximations.

It can be observed that stabilizing the solutions yields much better results for the coarser meshes and that both approaches converge to the same results for low local Péclet numbers. However, in this particular example, since the SUPG approximations are not obtained using an optimal stabilitzation parameter, for very fine meshes, the SUPG approximation does not perform better than the Galerkin approximation and thus the bounds for the output are also a little bit worse. Figure 8 displays the primal and adjoint solutions obtained in the final meshes along with the final adapted meshes obtained for both the Galerkin and SUPG approaches. It can be observed that the meshes are refined in the areas where either the primal or adjoint solutions present larger gradients and that both approaches provide very close results. 
stabilized SUPG finite element approximation

\begin{tabular}{c|c||ccc||ccc}
\hline \multicolumn{1}{l|}{} & \multicolumn{3}{c||}{ flux-free } & \multicolumn{3}{c}{ hybrid-flux } \\
\hline$n_{\mathrm{el}}$ & $s_{h}$ & $s^{l b}$ & $s^{u b}$ & $\Delta$ & $s^{l b}$ & $s^{u b}$ & $\Delta$ \\
\hline 300 & 0.407135 & -5.336663 & 6.048435 & 5.692549 & -11.895049 & 11.616651 & 11.755850 \\
348 & 0.429727 & -4.281018 & 4.983699 & 4.632359 & -10.633115 & 10.369201 & 10.501158 \\
418 & 0.428071 & -3.166066 & 3.868888 & 3.517477 & -9.588084 & 8.985694 & 9.286889 \\
516 & 0.421839 & -2.265427 & 2.983470 & 2.624448 & -7.666838 & 7.408345 & 7.537592 \\
610 & 0.403156 & -1.464917 & 2.228815 & 1.846866 & -5.698544 & 6.070066 & 5.884305 \\
735 & 0.401751 & -1.056960 & 1.829619 & 1.443289 & -4.794489 & 5.237296 & 5.015893 \\
918 & 0.395629 & -0.608291 & 1.377529 & 0.992910 & -3.717249 & 4.246143 & 3.981696 \\
1139 & 0.388709 & -0.326000 & 1.097425 & 0.711713 & -2.993937 & 3.696043 & 3.344990 \\
1347 & 0.386049 & -0.189252 & 0.957050 & 0.573151 & -2.588543 & 3.311109 & 2.949826 \\
1634 & 0.385730 & -0.030445 & 0.796235 & 0.413340 & -2.016893 & 2.736674 & 2.376783 \\
2017 & 0.382891 & 0.086123 & 0.677498 & 0.295688 & -1.641892 & 2.387876 & 2.014884 \\
2366 & 0.382033 & 0.142205 & 0.620629 & 0.239212 & -1.611950 & 2.359493 & 1.985721 \\
2830 & 0.381993 & 0.196499 & 0.565911 & 0.184706 & -1.330300 & 2.063420 & 1.696860 \\
3383 & 0.380941 & 0.247565 & 0.513481 & 0.132958 & -1.108488 & 1.852603 & 1.480546 \\
4032 & 0.380849 & 0.285735 & 0.474613 & 0.094439 & -1.022337 & 1.762936 & 1.392637 \\
4677 & 0.380305 & 0.306157 & 0.453796 & 0.073820 & -0.839117 & 1.580229 & 1.209673 \\
5456 & 0.380254 & 0.322223 & 0.438008 & 0.057893 & -0.731359 & 1.482723 & 1.107041 \\
6417 & 0.380200 & 0.336554 & 0.423612 & 0.043529 & -0.553114 & 1.311487 & 0.932301 \\
7347 & 0.380118 & 0.348275 & 0.411643 & 0.031684 & -0.326203 & 1.084242 & 0.705222 \\
8684 & 0.380082 & 0.355037 & 0.404833 & 0.024898 & -0.257523 & 1.015184 & 0.636353 \\
10264 & 0.379933 & 0.359460 & 0.400439 & 0.020490 & -0.213317 & 0.973382 & 0.593350 \\
12330 & 0.379947 & 0.364213 & 0.395691 & 0.015739 & -0.108349 & 0.867665 & 0.488007 \\
\hline
\end{tabular}

Table IV. Example 2: bounds for an apative $h$-refinement procedure obtained from SUPG approximations.

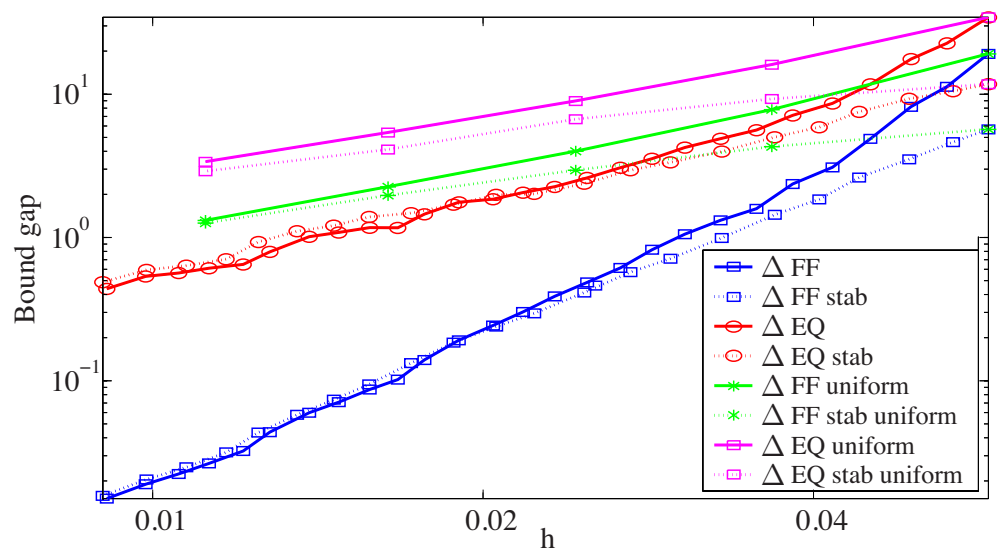

Figure 7. Example 2: convergence of the half bound gap for an adaptive $h$-refinement procedure obtained from standard Galerkin finite element approximations and SUPG approximations. Comparison with the results for the uniform mesh refinement.

\subsection{Example 3: inner shockfront and boundary layer}

The final example is a advection-diffusion problem posed on the unit square $\Omega=[0,1] \times[0,1]$ with $\nu=1, \sigma=0$ and a uniform velocity field $\boldsymbol{\alpha}=(300,150)$. The right-hand side is homogeneous, $f=0$ and on the whole boundary Dirichlet boundary conditions are given $u_{D}=1$ at the lower and 

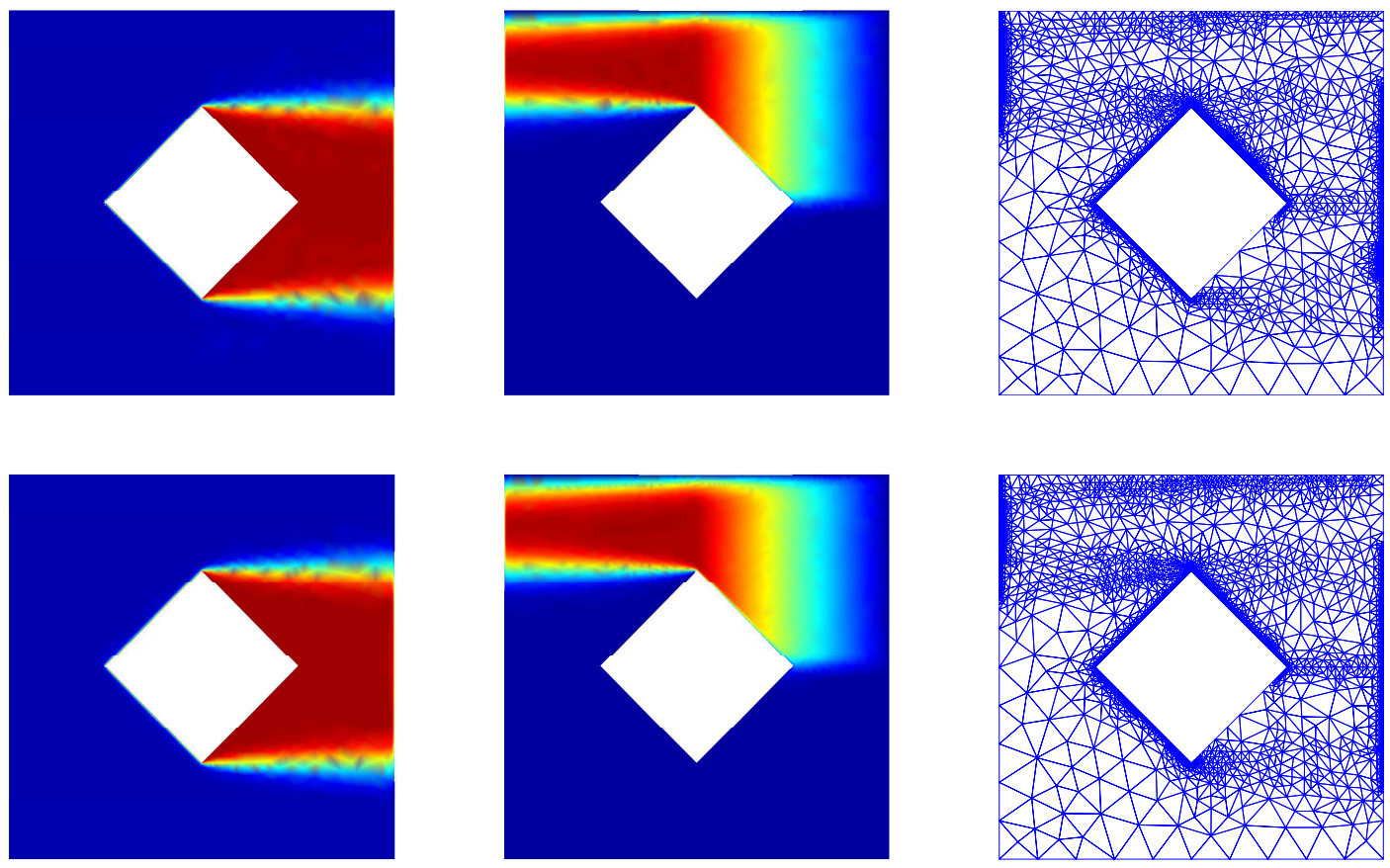

$\begin{array}{lllllllllllll}0 & 0.1 & 0.2 & 0.3 & 0.4 & 0.5 & 0.6 & 0.7 & 0.8 & 0.9 & 1\end{array}$

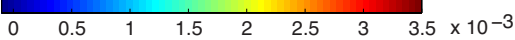

Figure 8. Example 2: Primal (left) and adjoint (center) solutions for the last meshes of the adaptive refinement obtained using the standard Galerkin finite element method (top) and the SUPG method (bottom). Final meshes consisting of 12126 elements for the Galerkin method (top-right) and 12330 for the SUPG method (bottom-right).

right boundary and $u_{D}=0$ elsewhere (see figure 9). This example has been presented in [28]. Due
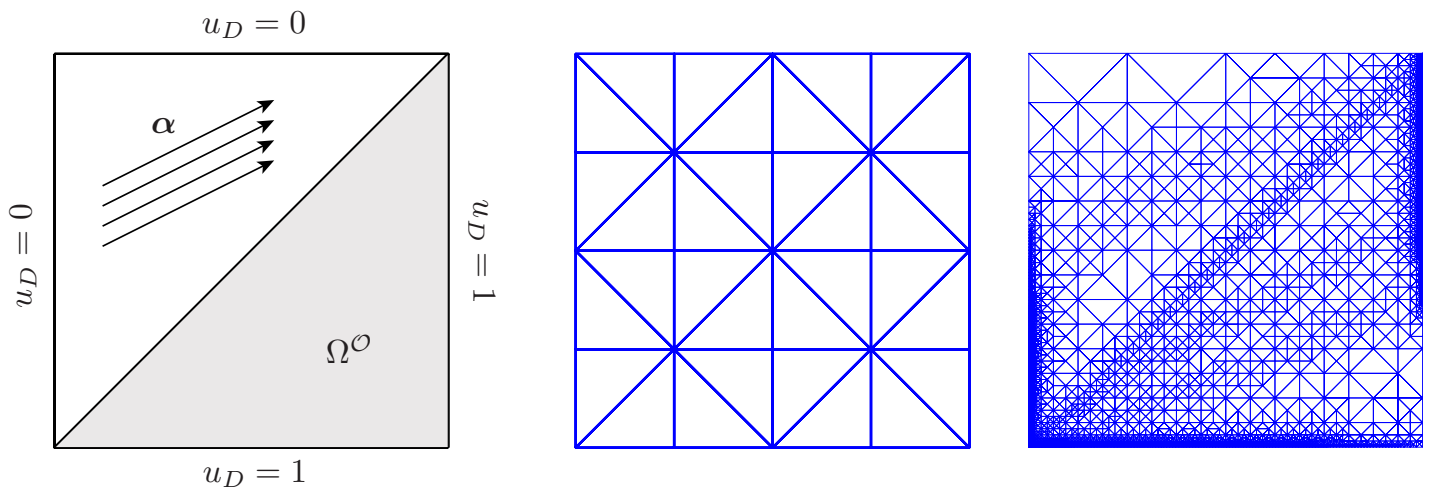

Figure 9. Example 3: Domain (left), initial mesh (both for the uniform and adaptive refinements) consisting of 32 triangular linear elements (middle) and final mesh of the adaptive procedure for the standard Galerkin approach consisting of 12524 elements.

to the velocity field and the distribution of the boundary conditions, an inner shockfront appears starting in the lower left corner and a boundary layer occurs at the right boundary, from $y=1 / 2$ to $y=1$. 
The quantity of interest is taken to be the integral of the solution over the lower right half square, namely

$$
\ell^{\mathcal{O}}(u)=\int_{\Omega^{\mathcal{O}}} u(x, y) \mathrm{d} \Omega
$$

which corresponds to $f^{\mathcal{O}}=1$ in $\Omega^{\mathcal{O}}$ and zero elsewhere. That is $\Omega^{\mathcal{O}}=\{(x, y) \in \Omega, x \geq y\}$ as can be seen in figure 9 . Both the primal and adjoint solutions obtained in the final mesh of the adaptive procedure are shown in figure 10.
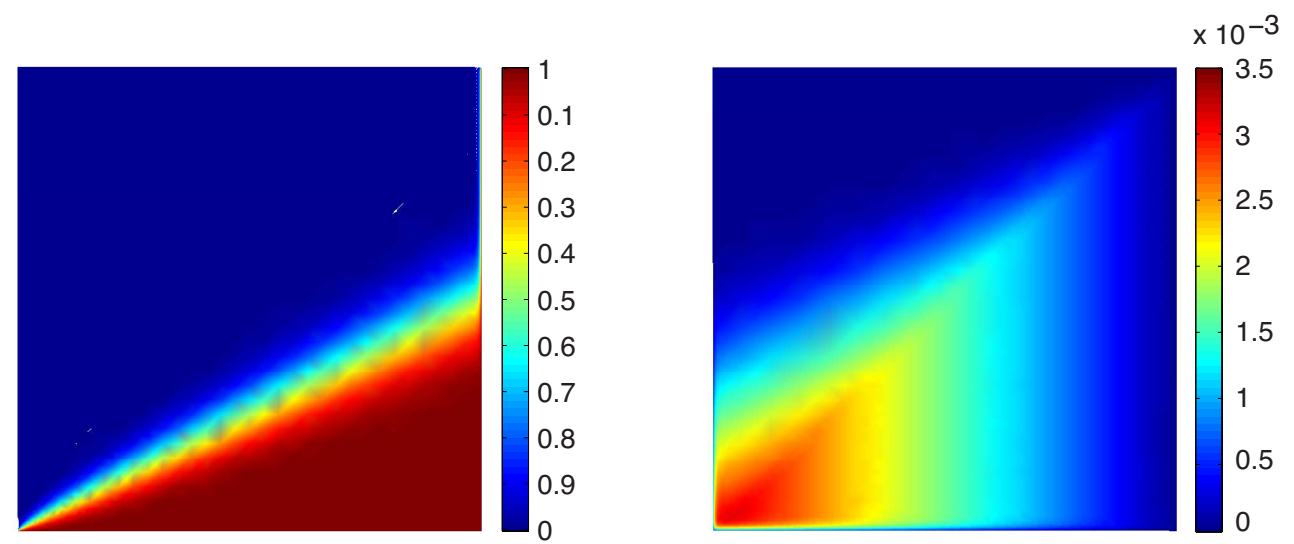

Figure 10. Example 3: Primal (left) and adjoint (right) solutions for the last mesh of the adaptive procedure.

The sensitivity of the proposed error estimation strategy is tested with respect to the definition of the stabilization parameter. Although the optimal selection of this parameter is not addressed in this paper, since the choice of the stabilization significantly influences the quality of the discrete solution three different choices for the stabilization parameter have been considered here to be able to compare the efficiency of the bounds for the quantity of interest.

The first choice of the stabilization parameter is the stabilization parameter used in the two first examples given in equations (33) and (34), denoted by $\tau_{k}^{1}$. Note that in this particular example, the stabilization parameter for the primal and adjoint problem coincide since the velocity field is divergence free. This stabilization parameter is compared with the well known expression $h_{k} /\left(2|\boldsymbol{\alpha}|_{k}\right)\left(\operatorname{coth}\left((P e)_{k}\right)-1 /(P e)_{k}\right)$. In order to compute the previous expression, two different choices for the element size are used: the smallest edge side of the triangle, $h_{k}^{1}$, and the diameter of the element $\Omega_{k}$ in the direction of the advection field $\alpha, h_{k}^{2}$, see [24]. These two different expressions to compute the element size yield two different choices of the stabilization parameter, denoted by $\tau_{k}^{2}$ and $\tau_{k}^{3}$ respectively.

The quality of the bounds is analyzed for an adaptive refinement. A series of adapted meshes is produced by subdividing at each step $10 \%$ of the elements, those with the larger contributions to the half bound gap, until $\Delta<0.002$. The adaptive procedure is guided by the indicators (local half bound gap) provided by the strict flux-free error estimate. The results for the hybrid-flux method are not reported since, as in the previous examples, its performance is much worse than the flux-free method.

The initial mesh of 32 elements certifies a wide interval for the quantity of interest $s=$ $40.085 \pm 44.666$ using the standard Galerkin approach and $s_{1}=0.340 \pm 1.870, s_{2}=0.341 \pm 1.859$ and $s_{3}=0.312 \pm 1.777$ for the three different SUPG approximations (associated with $\tau_{k}^{1}, \tau_{k}^{2}$ and $\tau_{k}^{3}$ respectively). As it can be seen, in the initial mesh, there is a great difference between the nonstabilized formulations and the stabilized ones. The different choices of the stabilization parameter yield to similar results, the third one being the best one for this problem.

After remeshing, the bounds associated with the final mesh set a much narrower interval $s=$ $0.25793 \pm 0.00194=0.25793 \pm 0.75 \%$ (for the standard Galerkin approach for a mesh of 12524 elements), $s_{1}=0.25784 \pm 0.00191=0.25784 \pm 0.74 \%$ (for the SUPG approach for a mesh of 
12507 elements), $s_{2}=0.25784 \pm 0.00191=0.25784 \pm 0.74 \%$ (for the SUPG approach for a mesh of 12418 elements) and $s_{3}=0.25786 \pm 0.00187=0.25786 \pm 0.72 \%$ (for the SUPG approach for a mesh of 13280 elements).

The convergence of the bounds is shown in figure 11. Again for the coarser meshes, the use of stabilization provides better results, and as the meshes are refined, the half bound gap reduction provided by stabilization techniques becomes less important. It can also be appreciated that once the finite element meshes are fine enough, there is no big difference between Galerkin and SUPG.
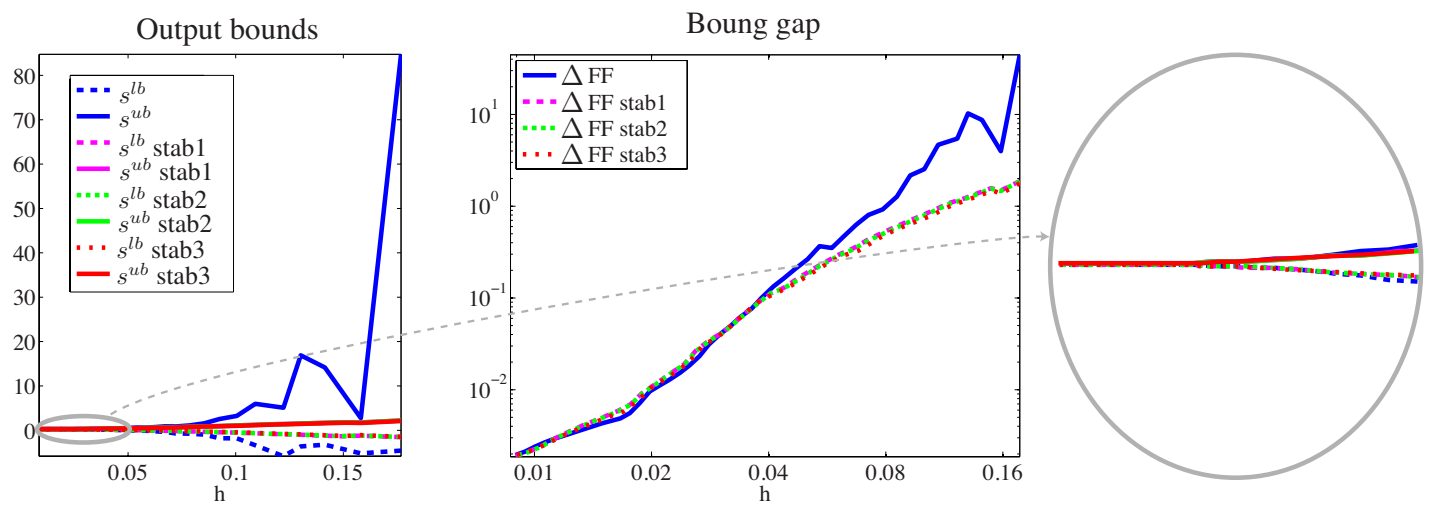

Figure 11. Example 3: Series of adapted $h$-refined. Bounds (left) and their convergence (right) for the standard Galerkin approach and the SUPG approach for the three different choices of the stabilization parameter.

The final mesh of the adaptive procedure for the standard Galerkin approach is shown in figure 10. The final meshes associated to the stabilized approaches are not shown since they are practically identical to the one obtained using the standard Galerkin approach. Thus stabilized techniques are well suited to drive goal-oriented adaptive procedures. It is worth noting for this quantity of interest, the meshes are refined mainly in the boundary layer and that there is no need to overly refine the interior shock front to obtain accurate approximations of the quantity of interest.

\section{CONCLUSIONS}

A simple and effective extension of guaranteed goal-oriented implicit residual estimators to stabilized methods has been presented. Both hybrid-flux and flux-free strategies have been extended to be able to deal with stabilized approximations of the exact solution. Thus, this paper introduces two new techniques to compute strict upper and lower bounds for functional outputs from stabilized approximations.

The proposed strategies are an extension of the flux-free technique presented in [11] and the hybrid-flux technique presented in [10]. The flux-free estimates yield much sharper bounds than the hybrid-flux approach both for the stabilized and non-stabilized approaches.

The presented strategies are only valid, as they stand, for stabilization techniques which may be rewritten in the form (4) including the widely used SUPG and SU techniques. Although the performance of the estimates is only shown for the SUPG method, the results presented herein for the SUPG methods using the hybdrid-flux equilibration are in very good agreement with the results presented in [19] for the SU method using also a modification of the hybrid-flux method. No significant differences are observed between the performance of the estimates due to the choice of the stabilization technique. Thus, it is expected that the fact of selecting one among the different stabilization techniques represented by the form (4) does not affect the performance of the estimates.

As shown in [11] the bounds for the quantity of interest are not robust with respect to the advection parameter, since the effectivities of the bounds deteriorate as the advection term becomes 
dominant. In this work, sharper bounds which alleviate this behavior have been obtained combining stabilization techniques along with goal-oriented adaptivity. Obtaining robust bounds for quantities of interest in the context of advection-dominated problems is still and open research topic, this work being a first contribution.

Finally, the indicators provided by the error estimators are well suited to guide goal-oriented adaptive procedures. It has also been observed that when adaptivity is used, special care should be taken when defining the stabilization parameter to yield stabilized discrete approximations better than the standard Galerkin approximations.

\section{REFERENCES}

1. Parés N, Díez P, Huerta A. Exact bounds for linear outputs of the advection-diffusion-reaction equation using flux-free error estimates. SIAM J. Sci. Comput. 2009; 31(4):3064-3089, doi:10.1137/080724356. URL http://dx.doi.org/10.1137/080724356.

2. Sauer-Budge AM, Bonet J, Huerta A, Peraire J. Computing bounds for linear functionals of exact weak solutions to Poisson's equation. SIAM J. Numer. Anal. 2004; 42(4):1610-1630 (electronic), doi:10.1137/S0036142903425045. URL http://dx.doi.org/10.1137/S0036142903425045.

3. Parés N, Bonet J, Huerta A, Peraire J. The computation of bounds for linear-functional outputs of weak solutions to the two-dimensional elasticity equations. Comput. Methods Appl. Mech. Engrg. 2006; 195(4-6):406-429, doi: 10.1016/j.cma.2004.10.013. URL http://dx.doi.org/10.1016/j.cma.2004.10.013.

4. Xuan ZC, Parés N, Peraire J. Computing upper and lower bounds for the $J$-integral in two-dimensional linear elasticity. Comput. Methods Appl. Mech. Engrg. 2006; 195(4-6):430-443, doi:10.1016/j.cma.2004.12.031. URL http://dx.doi.org/10.1016/j.cma.2004.12.031.

5. Parés N, Díez P, Huerta A. Bounds of functional outputs for parabolic problems. II. Bounds of the exact solution. Comput. Methods Appl. Mech. Engrg. 2008; 197(19-20):1661-1679.

6. Ladevèze $P$, Chamoin L. Calculation of strict error bounds for finite element approximations of non-linear pointwise quantities of interest. Internat. J. Numer. Methods Engrg. 2010; 84(13):1638-1664, doi:10.1002/nme.2957. URL http://dx.doi.org/10.1002/nme.2957.

7. Panetier J, Ladevèze P, Chamoin L. Strict and effective bounds in goal-oriented error estimation applied to fracture mechanics problems solved with XFEM. Internat. J. Numer. Methods Engrg. 2010; 81(6):671-700.

8. Moitinho de Almeida JP, Almeida Pereira OJB. Upper bounds of the error in local quantities using equilibrated and compatible finite element solutions for linear elastic problems. Comput. Methods Appl. Mech. Engrg. 2006; 195(4-6):279-296, doi:10.1016/j.cma.2004.09.012. URL http://dx.doi.org/10.1016/j.cma.2004.09.012.

9. Ern A, Stephansen AF, Vohralík M. Guaranteed and robust discontinuous Galerkin a posteriori error estimates for convection-diffusion-reaction problems. J. Comput. Appl. Math. 2010; 234(1):114-130, doi:10.1016/j.cam.2009. 12.009. URL http://dx.doi.org/10.1016/j.cam.2009.12.009.

10. Sauer-Budge AM, Peraire J. Computing bounds for linear functionals of exact weak solutions to the advection-diffusion-reaction equation. SIAM J. Sci. Comput. 2004; 26(2):636-652 (electronic), doi:10.1137/ S1064827503427121. URL http://dx.doi.org/10.1137/S1064827503427121.

11. Parés N, Díez P, Huerta A. Subdomain-based flux-free a posteriori error estimators. Comput. Methods Appl. Mech. Engrg. 2006; 195(4-6):297-323, doi:10.1016/j.cma.2004.06.047. URL http://dx.doi.org/10.1016/j.cma.2004.06.047.

12. Donea J, Huerta A. Finite Element Methods for Flow Problems. Wiley-Interscience [John Wiley \& Sons]: Chichester, 2003.

13. Ladevèze P, Leguillon D. Error estimate procedure in the finite element method and applications. SIAM J. Numer. Anal. 1983; 20(3):485-509, doi:10.1137/0720033. URL http://dx.doi.org/10.1137/0720033.

14. Ainsworth M, Oden JT. A posteriori error estimation in finite element analysis. Pure and Applied Mathematics (New York), Wiley-Interscience [John Wiley \& Sons]: New York, 2000.

15. Díez P, Parés N, Huerta A. Error Estimation and Quality Control, vol. 3, chap. 144. WileyInterscience [John Wiley \& Sons], 2010; 1725-1734, doi:10.1002/9780470686652.eae164. URL http://dx.doi.org/10.1002/9780470686652.eae164.

16. Oden JT, Prudhomme S. Goal-oriented error estimation and adaptivity for the finite element method. Comput. Math. Appl. 2001; 41(5-6):735-756, doi:10.1016/S0898-1221(00)00317-5. URL http://dx.doi.org/10.1016/s0898-1221(00)00317-5.

17. Becker R, Rannacher R. An optimal control approach to a posteriori error estimation in finite element methods. Acta Numer. 2001; 10:1-102, doi:10.1017/S0962492901000010. URL http://dx.doi.org/10.1017/s0962492901000010.

18. Parés N, Díez P, Huerta A. Bounds of functional outputs for parabolic problems. I. Exact bounds of the discontinuous Galerkin time discretization. Comput. Methods Appl. Mech. Engrg. 2008; 197(19-20):1641-1660.

19. Machiels L, Patera A, Peraire J, Maday Y. A general framework for finite element a posteriori error control: application to linear and nonlinear convection-dominated problems. M.J. Baines, Editor, Numerical Methods for Fluid Dynamics, ICFD, Oxford, UK, 1998.

20. Parés N, Santos H, Díez P. Guaranteed energy error bounds for the Poisson equation using a flux-free approach: solving the local problems in subdomains. Internat. J. Numer. Methods Engrg. 2009; 79(10):1203-1244, doi: 10.1002/nme.2593. URL http://dx.doi.org/10.1002/nme.2593. 
21. Paraschivoiu M, Peraire J, Patera AT. A posteriori finite element bounds for linear-functional outputs of elliptic partial differential equations. Comput. Methods Appl. Mech. Engrg. 1997; 150(1-4):289-312, doi: 10.1016/S0045-7825(97)00086-8. URL http://dx.doi.org/10.1016/s0045-7825(97)00086-8, symposium on Advances in Computational Mechanics, Vol. 2 (Austin, TX, 1997).

22. Shakib F, Hughes TJR, Johan Z. A new finite element formulation for computational fluid dynamics. X. The compressible Euler and Navier-Stokes equations. Comput. Methods Appl. Mech. Engrg. 1991; 89(1-3):141-219, doi:10.1016/0045-7825(91)90041-4. URL http://dx.doi.org/10.1016/0045-7825(91)90041-4, second World Congress on Computational Mechanics, Part I (Stuttgart, 1990).

23. Hauke G. A simple subgrid scale stabilized method for the advection-diffusion-reaction equation. Comput. Methods Appl. Mech. Engrg. 2002; 191(27-28):2925-2947, doi:10.1016/S0045-7825(02)00217-7. URL http://dx.doi.org/10.1016/S0045-7825(02)00217-7.

24. John V, Knobloch P. On spurious oscillations at layers diminishing (SOLD) methods for convection-diffusion equations. I. A review. Comput. Methods Appl. Mech. Engrg. 2007; 196(17-20):2197-2215, doi:10.1016/j.cma. 2006.11.013. URL http://dx.doi.org/10.1016/j.cma.2006.11.013.

25. Harari I, Frey S, Franca LP. A note on a recent study of stabilized finite element computations for heat conduction. Comput. Mech. 2002; 28(1):63-65, doi:10.1007/s00466-001-0270-2. URL http://dx.doi.org/10.1007/s00466-001-0270-2.

26. Codina R. On stabilized finite element methods for linear systems of convection-diffusion-reaction equations. Comput. Methods Appl. Mech. Eng. 2000; 188(1-3):61-82, doi:10.1016/S0045-7825(00)00177-8.

27. Knobloch P. On the definition of the SUPG parameter. Electron. Trans. Numer. Anal. 2008; 32:76-89.

28. Papastavrou A, Verfürth R. A computational comparison of a posteriori error estimators for convection-diffusion problems. Computational mechanics (Buenos Aires, 1998). Centro Internac. Métodos Numér. Ing.: Barcelona, 1998; CD-ROM file. 